\title{
GLOBAL BIFURCATION PROBLEMS ASSOCIATED WITH $k$-HESSIAN OPERATORS
}

\author{
JON JACOBSEN
}

\begin{abstract}
In this paper we study global bifurcation phenomena for a class of nonlinear elliptic equations governed by the $h$-Hessian operator. The bifurcation phenomena considered provide new methods for establishing existence results concerning fully nonlinear elliptic equations. Applications to the theory of critical exponents and the geometry of $k$-convex functions are considered. In addition, a related problem of Liouville-Gelfand type is analyzed.
\end{abstract}

\section{Introduction}

Let $\Omega$ be a domain in $\mathbb{R}^{n}$. If $k \in\{1, \ldots, n\}$ and $u \in C^{2}(\Omega)$, then the $k$-Hessian operator is defined by

$$
S_{k}\left(D^{2} u\right)=S_{k}\left(\lambda\left[D^{2} u\right]\right)=\sum_{1 \leq i_{1}<\ldots<i_{k} \leq n} \lambda_{i_{1}} \ldots \lambda_{i_{k}},
$$

where $\lambda[r]=\left(\lambda_{1}, \ldots, \lambda_{n}\right)$ denotes the eigenvalues of the symmetric matrix $r$ and $S_{k}$ is the $k^{t h}$ elementary symmetric polynomial in $n$ variables. Notice that $S_{1}\left(D^{2} u\right)=\Delta u$ and $S_{n}\left(D^{2} u\right)=\operatorname{det} D^{2} u$. Thus, the $k$-Hessian operators form a discrete collection of partial differential operators, which includes the Laplace and Monge-Ampère operators. In this framework, it is natural to think of the

\footnotetext{
1991 Mathematics Subject Classification. Primary 35J65; Secondary 35P30.

Key words and phrases. Global bifurcation, Monge-Ampère equations, $k$-Hessian equations, $k$-convex functions, Krein-Rutman, principal eigenvalue, critical exponents.

The author would like to express his gratitude to Professor Klaus Schmitt under whose guidance this work was carried out.
} 
Laplace and Monge-Ampère operator as connected by a family of operators, whose properties vary as $k$ varies. This connection raises many interesting questions concerning the $k$-Hessian operators.

The study of Monge-Ampère equations began with the early work of Monge [32] in 1784 and was continued by Ampère [1] in 1820. Motivated by questions from geometry, Monge undertook a study of equations of the form

$$
A\left(r t-s^{2}\right)+B r+C s+D t+E=0
$$

where the coefficients $A, B, C, D, E$ are functions of $x, y, u, p$, and $q$. Here $x$ and $y$ are the independent variables, $p=\partial u / \partial x$ and $q=\partial u / \partial y$ are the components of the gradient of $u$, and $r=\partial^{2} u / \partial x^{2}, s=\partial^{2} u / \partial x \partial y$, and $t=\partial^{2} u / \partial y^{2}$ are the elements of the Hessian matrix $D^{2} u$. Monge studied a transformation to convert (0.1) into a system of ordinary differential equations to which he then could apply integral methods. Ampère generalized the method of Monge to develop a method which transformed (0.1) into a system of first order partial differential equations. Although there were several further contributions to the theory (e.g. Boole [3] and De Morgan [11]), it was not until the work of Lie [26], [27] that the depth of the contributions of Monge and Ampère were realized. In fact, it seems to be Lie [27] who first used the terminology "Monge-Ampère" for equations of the form (0.1). The following relevant quote concerning the work of Monge and Ampère is taken from the preface of Goursat's text [16, p. vii]: "On n'a pas assez remarqué, il me semble, ces profondes recherches du grand géomètre, où sont employées des transformations de contact tout à fait générales, un demisiècle avant les travaux de M. Sophus Lie."

During this century the Monge-Ampère operator has continued to enjoy a great deal of investigation (see e.g. [31], [25], [36], [2], [5], [15], [28], [19]), in particular for its applications to problems from geometry. Beginning with the work of Krylov [23] and Caffarelli, Nirenberg, and Spruck [6], there has been considerable study of the general $k$-Hessian operators (e.g. [44], [45], [47]-[49]).

The focus of this paper is to study global bifurcation phenomena for the class of $k$-Hessian operators and some consequences thereof. In addition to being of independent interest, the bifurcation phenomena studied here provide new methods for establishing various existence results concerning fully nonlinear elliptic equations.

The main equation of study is defined by

$$
\begin{cases}S_{k}\left(D^{2} u\right)=f(\lambda, u) & x \in \Omega, \\ u=0 & x \in \partial \Omega\end{cases}
$$


where $\Omega$ is a strictly $(k-1)$-convex domain in $\mathbb{R}^{n}$. In addition to considering "powerlike" growth conditions on $f$, we shall also consider the case of exponential growth, in which case (0.2) may be thought of as a Liouville-Gelfand type problem.

An interesting case where an equation of the form (0.2) arises with $k=n$, is in the study of evolution problems for nonparametric surfaces with speed depending on Gauss curvature. In [33], Oliker considers equations of the form

$$
\begin{cases}u_{t}=\frac{D^{2} u}{\left(1+|D u|^{2}\right)^{\beta}} & \text { in }(0, \infty) \times \Omega, \\ u(x, t) \text { is strictly convex } & \text { for each } t \geq 0, \\ u(x, t)=0 & \text { in }[0, \infty) \times \partial \Omega,\end{cases}
$$

for $\beta \geq 0$ a constant. Geometrically, the graphs of $u(x, t)$ may be thought of as a family of hypersurfaces evolving in $\mathbb{R}^{n+1}$ with a fixed boundary. For $\beta=(n+1) / 2$, the normal speed of a point $(x, u(x, t))$ is equal to the Gauss curvature of the graph at this point. By first looking for self-similar solutions to $(0.3)$ of the form $u(x, t)=\phi(t) \psi(x)$ in the case $\beta=0$, Oliker shows that the function $\psi$ would satisfy the Monge-Ampère equation

$$
\begin{cases}\operatorname{det} D^{2} \psi=|\delta \psi| & x \in \Omega, \\ \psi=0 & x \in \partial \Omega,\end{cases}
$$

for $\delta=1 /(n-1)$. Oliker then establishes the existence of a unique solution to (0.4) by sharpening some of the previously known a priori estimates [5], [7], [36] of the $C^{3}$-norm for the solution $\psi$. This solution is then used to analyze the asymptotic behavior for the solutions to $(0.3)$ when $\beta>0$. In particular, sharp estimates for the rate at which $u(x, t) \rightarrow 0$ as $t \rightarrow \infty$ in terms of this solution are given.

Another motivating example of equations of the form (0.2) are the so-called Liouville-Gelfand problems defined by

$$
\begin{cases}-S_{k}\left(D^{2} u\right)=\lambda e^{u} & x \in \Omega, \\ u=0 & x \in \partial \Omega .\end{cases}
$$

The classical Liouville-Gelfand problem is concerned with positive solutions to (0.5) in the case $k=1$, where $S_{1}\left(D^{2} u\right)=\Delta u$. In this case, if $\Omega=B_{R}(0)$ is the open ball of radius $R$ centered at the origin in $\mathbb{R}^{n}$, then by the well-known results of Gidas-Ni-Nirenberg [14], all positive solutions to (0.5) are radially symmetric; hence (0.5) becomes the ordinary differential equation

$$
\begin{cases}-u^{\prime \prime}-\frac{n-1}{r} u^{\prime}=\lambda e^{u} & r \in(0, R), \\ u>0 & r \in[0, r), \\ u^{\prime}(0)=u(R)=0 . & \end{cases}
$$


This problem was studied by Liouville [30] in the case $n=1$, Bratu [4] in the case $n=2$, and later, Gelfand [13] for higher dimensions. Of particular interest is the relationship between the space dimension and multiplicity results for (0.6) first observed by Joseph and Lundgren [20]. The results may be divided up into three cases, which we now briefly recall:

- (Case I) $n=1,2$. There exists a $\lambda^{*}>0$ such that (0.6) has exactly one solution for $\lambda=\lambda^{*}$ and exactly two solutions for $0<\lambda<\lambda^{*}$.

- (Case II) $3 \leq n \leq 9$. The continuum of solutions to (0.6) oscillates around $\lambda=2(n-2)$, with the amplitude of oscillations tending to zero, as $\|u\| \rightarrow \infty$.

- (Case III) $n \geq 10$. Equation (0.6) has a unique solution for each $\lambda \in$ $(0,2(n-2))$ and no solutions for $\lambda \geq 2(n-2)$.

In [9], the authors consider the Liouville-Gelfand problem associated with (0.5) for the $k$-Hessian operator when $k=n / 2$.

The purpose of this paper is to demonstrate how results concerning equations of the form (0.2) may be established using topological methods. In particular, using recent results due to Trudinger and Wang [43]-[45] for $k$-Hessian operators, we shall study (0.2) from the perspective of global bifurcation.

It is worth remarking that some of the results obtained in this paper overlap with earlier results of P. L. Lions [29], Wang [49], and Tso [46], [47]. Nevertheless, we believe that our point of view enjoys an inherent simplicity, may be useful for more general problems, and sheds some light into the nature of the geometry of $k$-convex functions.

This paper is organized as follows: in Section 1 we recall some fundamental results in the theory of $k$-Hessian operators. In Section 2 we develop an extension of the Krein-Rutman theorem to operators which are not necessarily linear or strongly positive but satisfy the "linear-like" properties of homogeneity and monotonicity. In Section 3 we shall discuss how the abstract results of Section 2 may be applied to the $k$-Hessian operators to establish the existence of a principal eigenvalue for the $k$-Hessian operator. This result was first established by Lions [29] for the Monge-Ampère operator and Wang [49] for the $k$-Hessian operator $(1<k<n)$. Here we see the results of Lions and Wang can be obtained in unison, as an application of the general results from Section 2. Interesting related results may be found in [9], where the authors establish the existence of a principal eigenvalue for a large class of ordinary differential operators which includes the radial cases for the $p$-Laplacian and $k$-Hessian.

In Section 4 we study bifurcation phenomena for equations of the form (0.2) for "powerlike" perturbations $f$. We divide the study of (0.2) into two distinct cases corresponding to sub/superlinear perturbations. 
In Section 5 we continue our study of global bifurcation phenomena associated with (0.2). The approach we use is to embed (0.2) into the one parameter family of equations

$$
\begin{cases}S_{k}\left(D^{2} u\right)=|\lambda u|^{k}+f(u) & x \in \Omega, \\ u=0 & x \in \partial \Omega,\end{cases}
$$

and consider the behavior of global bifurcation continua. In particular, we look for solutions to $(0.7)$ when $\lambda=0$. Here we demonstrate that in contrast to the Laplace operator, the Monge-Ampère operator does not have a critical exponent. This result was first established by Tso [46] using variational methods. Since the Laplace and Monge-Ampère operator are the first and last $k$-Hessian operator, respectively, it raises the natural question: For which values of $k$ does the $k$ Hessian operator have a critical exponent? We shall discuss this question and some related insight it gives into the geometry of $k$-convex functions.

In Section 6 we investigate the Liouville-Gelfand problem defined by (0.5) in the case of $k=n$. In particular, we shall demonstrate that, unlike the results of Joseph-Lundgren [20], the qualitative behavior of the solution continua to (0.5) do not depend on the space dimension $n$. We shall also discuss some results concerning (0.5) in the cases $1<k<n$.

The main results of the paper are as follows:

TheOREM 0.1. Let $E$ contain a cone $K$. Let $A: E \rightarrow K$ be a completely continuous operator with $\left.A\right|_{K}: K \rightarrow K$ homogeneous, monotone, and strong. Furthermore, assume there exists $w, A(w) \in \operatorname{Im}(A) \cap K \backslash\{0\}$. Then there exists a constant $\lambda_{0}>0$ with the following properties:

(1) There exists $u \in K \backslash\{0\}$, with $u=\lambda_{0} A(u)$.

(2) If $v \in K \backslash\{0\}$ and $\lambda>0$ such that $v=\lambda A(v)$, then $\lambda=\lambda_{0}$.

TheOREM 0.2. Let $\Omega$ be a strictly $(k-1)$-convex domain. Then there exists a unique positive constant $\lambda_{0}=\lambda_{0}(k, \Omega)$ such that the $k$-Hessian equation

$$
\begin{cases}S_{k}\left(D^{2} u\right)=\left|\lambda_{0} u\right|^{k} & x \in \Omega, \\ u=0 & x \in \partial \Omega,\end{cases}
$$

admits a nontrivial admissible solution $u$. We call $\lambda_{0}$ the eigenvalue for the $k$-Hessian operator associated with the domain $\Omega$. Moreover, the following statements are true:

(1) Let $u$ be a nontrivial solution to (0.8). If $v$ is any other nontrivial admissible solution to (0.8), then $v=\theta u$, for some $\theta>0$.

(2) If $\Omega^{\prime} \subset \Omega$, both strictly $(k-1)$-convex, then the eigenvalue associated with the domain $\Omega^{\prime}$ is strictly greater than the eigenvalue associated with the domain $\Omega$. 
THEOREM 0.3. The point $(\mu, 0)$ is a bifurcation point for the equation

$$
\begin{cases}S_{k}\left(D^{2} u\right)=\lambda\left(|u|^{k}+g_{0}(u)\right) & x \in \Omega, \\ u=0 & x \in \partial \Omega,\end{cases}
$$

if and only if $|\mu|=\lambda_{0}$. Furthermore, there exists an unbounded continuum $\mathcal{C}^{+}$ (resp. $\left.\mathcal{C}^{-}\right)$of nontrivial solutions which bifurcates from $\left(\lambda_{0}, 0\right)\left(\right.$ resp. $\left.\left(-\lambda_{0}, 0\right)\right)$ and lies in the strip $\left\{(\lambda, u): 0 \leq \lambda \leq \lambda_{0}\right\}$ (resp. $\left.\left\{(\lambda, u):-\lambda_{0} \leq \lambda \leq 0\right\}\right)$.

ThEOREM 0.4. The constant $\mu$ is an asymptotic bifurcation value for

$$
\begin{cases}S_{k}\left(D^{2} u\right)=\lambda\left(|u|^{k}+g_{\infty}(u)\right) & x \in \Omega, \\ u=0 & x \in \partial \Omega,\end{cases}
$$

if and only if $|\mu|=\lambda_{0}$. Furthermore, there exists an unbounded continuum of nontrivial solutions which lies in the strip $\left\{(\lambda, u):-\lambda_{0} \leq \lambda \leq \lambda_{0}\right\}$.

TheOREM 0.5. Let $\Omega \subset \mathbb{R}^{n}$ be a strictly convex, bounded domain. Then the Monge-Ampère equation

$$
\begin{cases}\operatorname{det} D^{2} u=|\delta u|^{p} & x \in \Omega, \\ u=0 & x \in \partial \Omega,\end{cases}
$$

has a nontrivial admissible solution for all $p \neq n, p \geq 0$, and $\delta \in \mathbb{R}$. In the case $p<n$, the solution is unique.

Theorem 0.6. Let $k \in\{1, \ldots, n\}, g(u)=|\delta u|^{p}$ for some $0 \leq p<k, \delta \in \mathbb{R}$, and suppose $\Omega$ is a strictly $(k-1)$-convex domain in $\mathbb{R}^{n}$. Then there exists a global continuum of nontrivial solutions to the $k$-Hessian equation

$$
\begin{cases}S_{k}\left(D^{2} u\right)=|\lambda u|^{k}+g(u) & x \in \Omega, \\ u=0 & x \in \partial \Omega,\end{cases}
$$

which crosses the $\lambda=0$ axis nontrivially. Therefore, the equation

$$
\begin{cases}S_{k}\left(D^{2} u\right)=|\delta u|^{p} & x \in \Omega, \\ u=0 & x \in \partial \Omega,\end{cases}
$$

has a nontrivial $k$-convex solution for all $0<p<k$, and $\delta \in \mathbb{R}$. Moreover, the solution is unique.

TheOREM 0.7. There exists $\lambda^{*}>0$ such that the equation

$$
\begin{cases}\operatorname{det} D^{2} u=\lambda e^{-u} & x \in \Omega, \\ u=0 & x \in \partial \Omega,\end{cases}
$$

has at least two solutions for all $\lambda \in\left(0, \lambda^{*}\right)$. Furthermore, there exists $\bar{\lambda}>0$ such that (10) has no solution for $\lambda>\bar{\lambda}$. 


\section{Preliminaries}

For a given convex function $u$, the $n$-Hessian operator gives rise to a Borel measure $\mu$ defined by

$$
\mu_{n}[e]=\int_{\chi_{u}(e)} 1 d x=\left|\chi_{u}(e)\right|
$$

where $\chi_{u}$ is the normal mapping (subdifferential) associated with the convex function $u[2]$. In this section we discuss the generalization of this measure to the intermediate $k$-Hessian operators, due to Trudinger and Wang [44], [45]. The key idea is to introduce the class of $k$-convex functions, for $k \in\{1, \ldots, n\}$. Important contributions to this theory may be found in the recent papers [6], [42]-[44], [10].

1.1. $k$-convex functions. For $k \in\{1, \ldots, n\}$, let $\Gamma_{k}$ be the component of $\left\{\lambda \in \mathbb{R}^{n}: S_{k}(\lambda)>0\right\} \subset \mathbb{R}^{n}$ containing the positive cone $\Gamma^{+}=\left\{\lambda \in \mathbb{R}^{n}: \lambda_{i}>0\right.$, $i=1, \ldots, n\}$. The set $\Gamma_{k}$ is a convex cone, with vertex at the origin, and is equal to the set $\left\{\lambda \in \mathbb{R}^{n}: S_{j}(\lambda)>0, j=1 \ldots, k\right\}$. Moreover, the chain of inclusions $\Gamma^{+}=\Gamma_{n} \subset \ldots \subset \Gamma_{k+1} \subset \Gamma_{k} \subset \ldots \subset \Gamma_{1}$ holds.

The cones $\Gamma_{k}$ are the key to defining $k$-convex functions.

Definition 1.1. Let $k \in\{1, \ldots, n\}$ and let $\Omega$ be a bounded domain in $\mathbb{R}^{n}$. A function $u \in C^{2}(\Omega)$ is called $k$-convex (uniformly $k$-convex) in $\Omega$ if $\lambda\left[D^{2} u\right] \in$ $\bar{\Gamma}_{k}\left(\Gamma_{k}\right)$ for each $x \in \Omega$. Equivalently, $u$ is $k$-convex if $S_{j}\left(D^{2} u\right) \geq 0(>0)$ in $\Omega$, for each $j=1, \ldots, k$.

For example, 1-convex functions satisfy $\Delta u \geq 0$, hence are subharmonic. Similarly, $n$-convex functions satisfy

$$
\operatorname{det} D^{2} u \geq 0, \ldots, S_{k}\left(\lambda\left[D^{2} u\right]\right) \geq 0, \ldots, \Delta u \geq 0
$$

hence are convex. By using the notion of viscosity solutions these notions may be readily extended to include continuous functions.

Definition 1.2. A continuous function $u: \Omega \rightarrow(-\infty, \infty)$ is called $k$-convex in $\Omega$, if $S_{k}\left(D^{2} u\right) \geq 0$ in the viscosity sense.

We shall denote the class of $k$-convex functions on $\Omega$ by $\Phi^{k}(\Omega)$. As above, a function $u \in C(\Omega)$ is 1-convex if and only if it is subharmonic and $n$-convex if and only if it is convex. It is shown in [44] that for $k>n / 2, k$-convex functions are in fact Hölder continuous, with exponent $\alpha=2-n / k>0$, generalizing the well-known fact that convex functions are Lipschitz continuous.

The following is a useful criterion for $k$-convex functions. 
Lemma $1.3([45])$. A function $u: \Omega \rightarrow(-\infty, \infty)$ is $k$-convex if and only if its restriction to any subdomain $\Omega^{\prime} \subset \subset \Omega$ is the limit of a monotone decreasing sequence in $C^{2}\left(\Omega^{\prime}\right) \cap \Phi^{k}\left(\Omega^{\prime}\right)$.

Whereas strict convexity is a natural condition for the domain when considering boundary value problems for Monge-Ampère equations, there is a corresponding notion of $k$-convexity for the domain which is natural for $k$-Hessian equations.

DeFinition 1.4 ( $k$-convex domain). Let $\Omega \subset \mathbb{R}^{n}$ be a bounded domain with $C^{2}$-boundary and let $k \in\{1, \ldots, n-1\}$. We say that the domain $\Omega$ is $k$-convex provided $\left(\kappa_{1}, \ldots, \kappa_{n-1}\right) \in \bar{\Gamma}_{k}$ for each $x \in \partial \Omega$, where $\kappa_{i}(i=1, \ldots, n-1)$ are the principal curvatures of $\partial \Omega$ at $x$. Equivalently, $\Omega$ is $k$-convex if $S_{j}\left(\kappa_{1}, \ldots, \kappa_{n-1}\right) \geq$ 0 for each $j=1, \ldots, k$ and for every $x \in \partial \Omega$.

Similarly, one defines a uniformly $k$-convex domain, by requiring the strict inequality $S_{j}\left(\kappa_{1}, \ldots, \kappa_{n-1}\right)>0$ to hold, for each $j=1, \ldots, k$, and for every $x \in \partial \Omega$. If $\kappa=\left(\kappa_{1}, \ldots, \kappa_{n-1}\right)$ is the vector of principal curvatures for $\partial \Omega$ at the point $x_{0}$, then the quantity $S_{j}\left(\kappa_{1}, \ldots, \kappa_{n-1}\right)$ is the $j$ th mean curvature of the boundary at $x_{0}$. Thus, a domain $\Omega$ will be $k$-convex provided the $j$ th mean curvatures of $\partial \Omega$ are nonnegative for each $j=1, \ldots, k$.

1.2. Symmetric polynomials. For the convenience of the reader, we collect some results concerning the symmetric functions acting on symmetric matrices. For $k$ fixed, with $1 \leq k \leq n$, we shall use the notation $S_{i j}(r) \doteq \partial S_{k}(r) / \partial r_{i j}$.

Proposition 1.5 ([49]). Let $r \in \mathbb{S}^{n \times n}$. If $\lambda[r] \in \Gamma_{k}$, then

(1) $\sum_{i=1}^{n} S_{i i}(r)=(n-k+1) S_{k-1}(r)$.

(2) $\left(S_{i j}(r)\right)$ is positive definite; i.e.

$$
\frac{\partial S_{k}(r)}{\partial r_{i j}} \xi_{i} \xi_{j}>0 \text { for all } \xi \in \mathbb{R}^{n} \backslash\{0\} \text { and } x \in \Omega \text {. }
$$

(3) $S_{k}(r)=\frac{1}{k} \sum_{i, j=1}^{n} r_{i j} S_{i j}(r)$.

Proof. Identity (1) may be verified directly. Property (2) is established in [6], where they show $\partial S_{k} / \partial \lambda_{i}>0$ for all $x \in \Omega$ and $1 \leq i \leq n$. Identity (3) follows from noticing that $S_{k}(r)$ is the sum of all $k \times k$ principal minors of $r$

$$
S_{k}(r)=\frac{1}{k !} \sum \delta\left(\begin{array}{c}
j_{1}, \ldots, j_{k} \\
i_{1}, \ldots, i_{k}
\end{array}\right) r_{i_{1} j_{1}} \cdot \ldots \cdot r_{i_{k} j_{k}}
$$

where $\delta\left(\begin{array}{l}j_{1}, \ldots, j_{k} \\ i_{1}, \ldots, i_{k}\end{array}\right)$ is the generalized Kronecker symbol defined to be 1 (resp. -1 ) if $i_{1}, \ldots, i_{k}$ are distinct and $\left(j_{1}, \ldots, j_{k}\right)$ is an even (resp. odd) permutation of $\left(i_{1}, \ldots, i_{k}\right)$; otherwise it is equal to 0 . Hence identity (3) follows from

$$
S_{i j}(r)=\frac{1}{(k-1) !} \sum \delta\left(\begin{array}{c}
i_{1}, \ldots, i_{k-1}, i \\
j_{1}, \ldots, j_{k-1}, j
\end{array}\right) r_{i_{1} j_{1}} \cdot \ldots \cdot r_{i_{k-1} j_{k-1}} .
$$


Let us introduce the "homogenized" function $F_{k}=\left(S_{k}\right)^{1 / k}$, and as above let $F_{i j}=\partial F_{k} / \partial r_{i j}$. One can check that the identity

$$
F_{i j}=\frac{1}{n} \frac{S_{i j}}{S_{k}^{(k-1) / k}}
$$

holds, in which case the matrix $\left(F_{i j}(r)\right)$ is positive definite whenever $\left(S_{i j}(r)\right)$ is positive definite and $S_{k}(r)>0$.

In [6], Caffarelli, Nirenberg, and Spruck show how an inequality due to Gårding [12] implies the following proposition.

Proposition 1.6. $F_{k}$ is concave on the set $\mathcal{A}=\left\{r \in \mathbb{S}^{n \times n}: \lambda[r] \in \Gamma_{k}\right\}$.

The properties above imply the following lemma for which we shall find use repeatedly in the sequel.

Lemma 1.7. Let $k \in\{1, \ldots, n\}$ and consider the linear operator $L$ defined by

$$
L=\sum_{i, j=1}^{n} F_{i j}\left(D^{2} w\right) D_{i j}
$$

Then $L$ is elliptic whenever $w \in \Phi^{k}(\Omega)$, and if $u, v \in \Phi^{k}(\Omega)$, then

$$
L(u-v) \geq F_{k}\left(D^{2} u\right)-F_{k}\left(D^{2} v\right)
$$

Proof. The inequality follows from the concavity of $F_{k}$ on $\Phi^{k}(\Omega)$ :

$$
L(u-v)=\sum_{i, j=1}^{n} F_{i j}\left(D^{2} w\right)\left(D_{i j} u-D_{i j} v\right) \geq F_{k}\left(D^{2} u\right)-F_{k}\left(D^{2} v\right) .
$$

1.3. Existence theorems. The class of $k$-convex functions is naturally related to the $k$-Hessian operators, in that, the operators $S_{k}\left(D^{2} u\right)$ are elliptic on the class of $k$-convex functions [6], and the Dirichlet problem

$$
\begin{cases}S_{k}\left(D^{2} u\right)=\psi(x) & x \in \Omega \\ u=\phi & x \in \partial \Omega\end{cases}
$$

has a unique $k$-convex solution $u \in C(\bar{\Omega})$ for any $\psi \in L^{p}(\Omega)$ with $p \geq n / 2 k$ and $\phi \in C(\bar{\Omega})$, if $\Omega$ is a uniformly $(k-1)$-convex domain. Furthermore, $u \in C^{\alpha}(\Omega)$ for any exponent $\alpha<1$, such that $\alpha \leq 2-n / k p$ [43, Theorem 1.1].

The main result of [45] is that for each $k$-convex function $u$, the $k$-Hessian operator defines a Borel measure $\mu_{k}$, and that the corresponding mapping $u \rightarrow$ $\mu_{k}[u]$ is weakly continuous. The resulting measure $\mu_{k}[u]$ is called the $k$-Hessian measure generated by $u$. 
Theorem 1.8 ([45]). Let $k \in\{1, \ldots, n\}$. Then, for any $u \in \Phi^{k}(\Omega)$, there exists a Borel measure $\mu_{k}[u]$ in $\Omega$ such that

(1) $\mu_{k}[u](e)=\int_{e} S_{k}\left(D^{2} u\right)$, for any Borel set $e \subset \Omega$, if $u \in C^{2}(\Omega)$, and

(2) if $\left\{u_{m}\right\}$ is a sequence converging locally in measure to a function $u \in$ $\Phi^{k}(\Omega)$ then $\mu_{k}\left[u_{m}\right] \rightarrow \mu_{k}[u] ;$ i.e.

$$
\int_{\Omega} g d \mu_{k}\left[u_{m}\right] \rightarrow \int_{\Omega} g d \mu_{k}[u]
$$

for all $g \in C(\Omega)$, with compact support.

From well-known properties of subharmonic functions (see e.g. [17]) we have the inclusions $\Phi^{k}(\Omega) \subset \Phi^{1}(\Omega) \subset L_{\text {loc }}^{1}(\Omega)$, and local convergence in measure is equivalent to convergence in $L_{\mathrm{loc}}^{1}(\Omega)$.

This generalization allows one to consider the corresponding Dirichlet problem (for measures) defined by

$$
\begin{cases}\mu_{k}[u]=\nu & \text { in } \Omega, \\ u=\phi & \text { on } \partial \Omega,\end{cases}
$$

in the class of $k$-convex functions. Presently, the most general theorem concerning (1.2) is due to Trudinger and Wang:

Theorem 1.9 (Trudinger-Wang, [44]). Let the domain $\Omega$ be uniformly $(k-$ 1)-convex. Suppose we are given the measure $\nu=\nu_{1}+\nu_{2}$, where $\nu_{1} \in L^{1}(\Omega)$ and $\nu_{2}$ has compact support. Then for any $\phi \in C(\bar{\Omega})$, there exists a unique $u \in \Phi^{k}(\Omega) \cap C(\bar{\Omega})$ satisfying (1.2), provided $k>n / 2$.

This theorem extends Theorem 1.1 of [43] in the case $k>n / 2$. In [44], the authors also establish a useful comparison principle, generalizing the well known cases $k=1$ and $k=n$ :

TheOREm 1.10 (Trudinger-Wang, [44]). Let $u, v \in \Phi^{k}(\Omega) \cap C(\bar{\Omega})$ satisfy

$$
\begin{cases}\mu_{k}[v] \leq \mu_{k}[u] & \text { in } \Omega, \\ u \leq v & \text { on } \partial \Omega,\end{cases}
$$

on the $(k-1)$-convex domain $\Omega$. Then $u \leq v$ in $\Omega$.

With this background material exposed, we are ready to investigate the $k$ Hessian equations. In light of the above remarks, unless otherwise stated, we shall assume throughout the rest of this paper that the domain $\Omega$ is a uniformly $(k-1)$-convex domain. 


\section{Krein-Rutman theory}

In this section we partially extend the classical Krein-Rutman theorem to completely continuous operators which are strong, monotone, and homogeneous. As an application of this theorem, we will prove the existence of a principal eigenvalue for the $k$-Hessian operator, in Section 3.

The Krein-Rutman theorem asserts that a strongly positive linear operator has a unique positive eigenfunction (of prescribed norm), and the corresponding eigenvalue is real and simple. The first theorem in this direction is the PerronFrobenius theorem:

Theorem 2.1 (Perron-Frobenius [18]). Let $A$ be an $n \times n$ matrix with strictly positive coefficients. Then $A$ has a positive eigenvalue $\lambda$, larger in magnitude than all the others, and the components of an associated eigenvector all have the same sign. Furthermore, the eigenvalue is simple.

Krein and Rutman extended this result to arbitrary dimensions by employing the theory of cones in Banach spaces:

Theorem 2.2 (Krein-Rutman [22]). Let $E$ contain a solid cone $K$. Let $L$ be a strongly positive linear compact operator. Then there exists a unique $\lambda_{0}>0$ such that the following statements are true:

(1) There exists $u \in \operatorname{Int}(K)$, with $u=\lambda_{0} L u$.

(2) If $(\lambda, v) \in \mathbb{R} \backslash\left\{\lambda_{0}\right\} \times E \backslash\{0\}$, with $v=\lambda L v$, then $v \notin K \cup\{-K\}$ and $\lambda_{0}<|\lambda|$.

One of the key ideas in this section is that the linearity assumption in the Krein-Rutman theorem may be replaced by a corresponding homogeneity and monotonicity assumption.

2.1. Existence of eigenvalues. Let $E$ be a real Banach space with a cone $K$. Recall that a cone $K$ introduces a partial order in $E$ by the relation

$$
u \leq v \text { if and only if } v-u \in K .
$$

By homogeneous, we shall mean positively homogeneous with degree 1. Furthermore, the term monotone shall refer to an operator that satisfies $A(x) \leq A(y)$ whenever $x \leq y$ in $E$.

The following theorem will be established in this section:

TheOrem 2.3. Let $A: E \rightarrow E$ be a completely continuous operator with $\left.A\right|_{K}: K \rightarrow K$ homogeneous and monotone. If there exists $w \in K \backslash\{0\}$ and a constant $\beta>0$ such that

$$
w \leq \beta A(w),
$$


then for each $R>0$, there exists $\lambda=\lambda(R)>0$ and $u \in K$, with $\|u\|=R$, such that

$$
u=\lambda A(u) .
$$

Furthermore, $\lambda \leq \beta$.

Thus, for each level set $\|u\|=R$ in $E$, there exists at least one solution pair $(\lambda, u)$ to $(2.3)$ such that $\|u\|=R$ and $\lambda \in(0, \beta]$. The constant $1 / \lambda$ will be called an eigenvalue of $A$, and the function $u$ will be called an eigenfunction of $A$ (corresponding to the eigenvalue $1 / \lambda$ ). Note that the condition that $A(E) \subset K$ implies $A$ is a positive operator. We also remark that if $A=0$, then (2.2) will not be satisfied for any $w \in K \backslash\{0\}$.

A key ingredient in the proof of Theorem 2.3 is the following lemma concerning bounds on possible values for $\lambda$ :

Lemma 2.4. Let $A: E \rightarrow E$ be a positive operator such that $\left.A\right|_{K}: K \rightarrow K$ is homogeneous and monotone. Assume there exists $w \in K \backslash\{0\}$ and a constant $\beta>0$ such that

$$
w \leq \beta A(w) .
$$

If $\lambda>0, \varepsilon>0$, and $u \in K$ are such that the equation

$$
u=\lambda A(u+\varepsilon w)
$$

holds, then $\lambda \leq \beta$.

Proof. Suppose $\lambda>0, \varepsilon>0$, and $u \in K$ are such that the equation

$$
u=\lambda A(u+\varepsilon w)
$$

holds. Since $\varepsilon>0$ and $w \in K$, it follows that $u \leq u+\varepsilon w$. Hence, by applying the monotonicity of $A$ to this inequality, we obtain $A(u) \leq A(u+\varepsilon w)$. Multiplying by $\lambda$ and employing (2.5) we arrive at the inequality

$$
\lambda A(u) \leq u .
$$

In a similar fashion (now requiring the homogeneity of $A$ ) we obtain the inequality

$$
\lambda \varepsilon A(w) \leq u
$$

Furthermore, by (2.4), we must have

$$
\frac{\lambda}{\beta} \varepsilon w \leq \lambda \varepsilon A(w) \leq u .
$$

Applying $A$ to this last inequality we obtain the inequality

$$
\frac{\lambda}{\beta} \varepsilon A(w) \leq A(u)
$$


Multiplying through by $\lambda$ and using (2.6) we have

$$
\frac{\lambda^{2}}{\beta} \varepsilon A(w) \leq u \text {. }
$$

One more application of (2.4) yields the inequality

$$
\left(\frac{\lambda}{\beta}\right)^{2} \varepsilon w \leq u .
$$

Applying $A$ repeatedly in this manner, we obtain (by induction) the inequality

$$
\left(\frac{\lambda}{\beta}\right)^{n} \varepsilon w \leq u, \quad \text { for each } n=1,2, \ldots .
$$

Multiplying each side of $(2.7)$ by $(\beta / \lambda)^{n}$ and recalling the definition of $\leq$ we see that

$$
\left(\frac{\beta}{\lambda}\right)^{n} u-\varepsilon w \in K, \quad \text { for all } n=1,2, \ldots \text {. }
$$

Therefore, if $\lambda>\beta$, then $(\beta / \lambda)^{n} u \rightarrow 0$ as $n \rightarrow \infty$ and (2.8) implies $-\varepsilon w \in K$, since $K$ is closed. However, since $K$ is a cone and $\varepsilon w \in K$, the fact that $-\varepsilon w \in K$ implies $w=0$, a contradiction, as by assumption $w \neq 0$. Therefore $\lambda \leq \beta$.

Notice that since $\beta$ is fixed, this estimate is independent of the value of $\varepsilon>0$ in (2.5). Using this lemma we may now establish Theorem 2.3.

Proof of Theorem 2.3. Fix $R>0$ and suppose there exists $w \in K \backslash\{0\}$ and $\beta>0$ such that $w \leq \beta A(w)$. Let $\widetilde{A}: E \rightarrow K$ denote the Dugundji extension of $A$ and consider the two parameter family of operators defined by

$$
f_{\varepsilon}(\lambda, u) \doteq u-\lambda \widetilde{A}(u+\varepsilon w) .
$$

For each fixed $\varepsilon>0$ and $\lambda>0$, the operator $f_{\varepsilon}(\lambda, \cdot): E \rightarrow E$ is a completely continuous perturbation of the identity. If $\lambda=0$, then the operator $f_{\varepsilon}(\lambda, \cdot)$ is the identity mapping; hence for the ball $B_{R}(0) \subset E$, the Leray-Schauder degree is defined and is given by

$$
d\left(f_{\varepsilon}(0, \cdot), B_{R}(0), 0\right)=d\left(\mathrm{id}, B_{R}(0), 0\right)=1 .
$$

On the other hand, if there exist constants $\varepsilon>0$ and $\lambda>0$ such that the equation

$$
f_{\varepsilon}(\lambda, u)=u-\lambda \widetilde{A}(u+\varepsilon w)=0,
$$

has a solution $u \in E$, then $u \in K$, since $\widetilde{A}(E) \subset K$. Therefore $\widetilde{A}=A$ in equation (2.10) and by Lemma 2.4, we may conclude that for any $\varepsilon>0$ and $\beta^{*}>\beta$ the equation

$$
u-\beta^{*} A(u+\varepsilon w)=0
$$


has no solutions in $E$. Thus the Leray-Schauder degree of $f_{\varepsilon}\left(\beta^{*}, \cdot\right)$ is defined, with

$$
d\left(f_{\varepsilon}\left(\beta^{*}, \cdot\right), B_{R}(0), 0\right)=0 .
$$

Since the degrees (2.9) and (2.11) are unequal, it follows from the homotopy invariance of the Leray-Schauder degree, that for each fixed $\varepsilon>0$, there exists $(\lambda, u) \in\left(0, \beta^{*}\right) \times \partial B_{R}(0)$ such that

$$
u=\lambda A(u+\varepsilon w) .
$$

In this way, we may construct a sequence of pairs $\left\{\left(\lambda_{k}, u_{k}\right)\right\}$, corresponding to $\left\{\varepsilon_{k}\right\}$, where $\varepsilon_{k} \rightarrow 0$ and $u_{k}$ solves (2.12) with $\lambda=\lambda_{k}$ and $\varepsilon=\varepsilon_{k}$. Note that by construction $\left\|u_{k}\right\|=R$ for each $k$. As the sequence $\left\{\lambda_{k}\right\} \subset(0, \beta]$, we may assume, by relabeling if necessary, that $\lambda_{k} \rightarrow \mu$ with $\mu \in[0, \beta]$. Since the sequence $\left\{u_{k}+\varepsilon_{k} w\right\}$ forms a bounded set in $E$, the complete continuity of $A$ implies the sequence $\left\{A\left(u_{k}+\varepsilon_{k} w\right)\right\}$ has a convergent subsequence. Once again, by relabeling if necessary, we may assume $A\left(u_{k}+\varepsilon_{k} w\right) \rightarrow z$. Note that $z \in K$ since $K$ is closed. Therefore, by (2.12), we may conclude the sequence $\left\{u_{k}\right\}$ contains a convergent subsequence, with $u_{k} \rightarrow \mu z \in K$. By the continuity of $A$ and (2.12), we may conclude that $\mu z=\mu A(\mu z)$. Since $\left\|u_{k}\right\|=R$ for all $k$, we have $\|\mu z\|=R$ and, in particular, $\mu z \in K \backslash\{0\}$. Thus, if we let $\lambda=\mu$ and $u=\mu z$, then the equation $u=\lambda A(u)$ is satisfied, with $0<\lambda \leq \beta, u \in K$, and $\|u\|=R$. Since $R>0$ was arbitrary, the proof is complete.

Note that for any level set $\|u\|=R>0$, the set of such constants $\lambda$, whose existence is guaranteed by the above theorem, is bounded below (away from 0 ).

There is an alternative way to view the proof of Theorem 2.3. For each fixed $\varepsilon>0$, we may apply Theorem 4.1 with $\lambda_{0}=0$, to conclude there exists at least one continuum

$$
\mathcal{C}_{\varepsilon} \subset \mathcal{S}_{\varepsilon}=\{(\lambda, u) \in[0, \infty) \times E:(\lambda, u) \text { solves }(2.10)\}
$$

which satisfies $\mathcal{C}_{\varepsilon} \cap(\{0\} \times E)=\{(0,0)\}$ and is unbounded in the space $[0, \beta] \times E$ (see Figure 2.1).

In particular, the continuum is unbounded in $E$, hence must meet each level set $\|u\|=R$. In this way, we may construct the sequence $\left\{\left(\lambda_{k}, u_{k}\right)\right\}$, with $\lambda_{k} \in$ $(0, \beta]$ and $\left\|u_{k}\right\|=R$. Then, arguing as before, we may extract a subsequence lying on the level $\|u\|=R$, and draw the same conclusions as above.

It is interesting to note that for $\varepsilon>0$ and $w \neq 0,(2.12)$ may not have the trivial branch of solutions. However, by homogeneity, $A(0)=0$; thus for $\varepsilon=0$, (2.12) does have the trivial branch of solutions $(\lambda, 0)$, and in fact, bifurcation from the trivial branch must occur by Theorem 2.3. Intuitively, one may imagine 


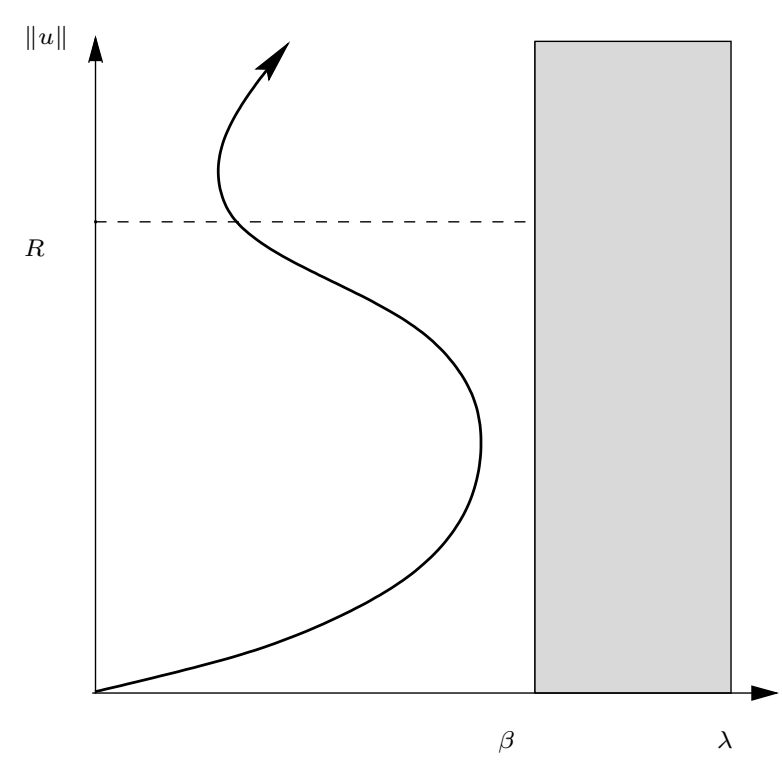

Figure 2.1. Continuum $C_{\varepsilon}$ for $f_{\varepsilon}(\lambda, u)=0$

the continua $\mathcal{C}_{\varepsilon}$ "flattening out" as $\varepsilon \rightarrow 0$. In particular, it is not unreasonable for $A$ to have many nonzero bifurcation values.

2.2. Strong operators. Solid cones and strongly positive operators play an important role in the study of semilinear elliptic problems. The operators studied here, however, cannot be put into this framework due to the nature of the nonlinearities. On the other hand, the full strength of strong positivity is not necessary to carry out arguments similar to those used in the semilinear case. With this in mind we introduce the following definition:

Definition 2.5 (strong operator). A positive operator $A: E \rightarrow E$ is called strong (relative to the cone $K$ ) if for all $u, v \in \operatorname{Im}(A) \cap K \backslash\{0\}$, there exist positive constants $\delta$ and $\gamma$ (which may depend on $u$ and $v$ ), such that

$$
u-\delta v \in K \quad \text { and } \quad v-\gamma u \in K .
$$

For example, if the cone $K$ is solid, any strongly positive operator is strong. For, if $u, v \in \operatorname{Int}(K)$, then $u-\delta v \in K$ for all $\delta>0$, sufficiently small.

The advantage of introducing strong operators is that we may work in cones which are not necessarily solid, and/or with operators which are not strongly positive. We shall see an example of an operator which is strong, but not strongly positive (relative to a fixed cone $K$ ) in Section 3.

The following lemma is useful for comparison arguments involving strong operators: 
LEMma 2.6. Let $A: E \rightarrow E$ be a strong operator and suppose $u, v \in \operatorname{Im}(A) \cap$ $K \backslash\{0\}$. Then there exists a maximal $\delta^{*}$ and $\gamma^{*}$ such that $u-\delta^{*} v \in K$ and $v-\gamma^{*} u \in K$.

Proof. Let $u, v \in \operatorname{Im}(A) \cap K \backslash\{0\}$ and suppose $u-\delta v \in K$ for all $\delta>0$. Then $u / \delta-v \in K$ for all $\delta>0$; hence $-v \in K$ as $K$ is closed. As $v \neq 0$, this contradiction implies the set of such $\delta$ is bounded above. Let $\Delta=\{\delta: u-\delta v \in$ $K\}$ and $\delta^{*}=\sup _{\Delta} \delta$. By the above argument, $\delta^{*}<\infty$ and we may conclude, by taking a sequence $\delta_{k} \rightarrow \delta^{*}$, that $u-\delta^{*} v \in K$ as $K$ is closed. The proof for $\gamma^{*}$ is identical.

In applications of Theorem 2.3, the key point is to find a constant $\beta>0$ and a cone element $w$ such that (2.2) holds. We next verify that if $A$ is a strong operator, then this condition is automatically satisfied. Furthermore, in the case that $A$ is strong, stricter conclusions may be drawn. Essentially, one can verify (2.2) holds for any strong operator, provided there exist nonzero elements $w, A(w) \in \operatorname{Im}(A) \cap K$. In the applications considered in later sections, this condition will follow from the stronger fact that the set $\mathcal{N}(A)=\{x \in E: A(x)=$ $0\}$ satisfies $\mathcal{N}(A)=\{0\}$. The corresponding generalization of Theorem 2.3 is as follows:

TheOrem 2.7. Let $E$ contain a cone $K$. Let $A: E \rightarrow E$ be a completely continuous operator with $\left.A\right|_{K}: K \rightarrow K$ homogeneous, monotone, and strong. Furthermore, assume that there exist nonzero elements $w, A(w) \in \operatorname{Im}(A) \cap K$. Then there exists a constant $\lambda_{0}>0$ with the following properties:

(1) There exists $u \in K \backslash\{0\}$, with $u=\lambda_{0} A(u)$.

(2) If $v \in K \backslash\{0\}$ and $\lambda>0$ such that $v=\lambda A(v)$, then $\lambda=\lambda_{0}$.

Proof. Assume $w, A(w) \in \operatorname{Im}(A) \cap K \backslash\{0\}$. Then the strength of $A$ implies that there exists $\delta>0$ such that $A(w)-\delta w \in K$. In terms of the partial order we have $\delta w \leq A(w)$. Therefore, by Theorem 2.3, there exists $\lambda_{0}>0$ and $u \in K \backslash\{0\}$ such that $u=\lambda_{0} A(u)$, establishing the first part of the theorem.

Let $(\lambda, v) \in(0, \infty) \times K \backslash\{0\}$, such that $v=\lambda A(v)$. The strength of $A$ combined with Lemma 2.6 implies that there exists $\delta^{*}>0$, maximal, such that

$$
u-\delta^{*} v \in K, \quad \text { i.e., } \quad \delta^{*} v \leq u \text {. }
$$

Using the monotonicity and homogeneity of $A$ we obtain $\delta^{*} A(v) \leq A(u)$. Equivalently,

Therefore

$$
\delta^{*} \frac{1}{\lambda} v \leq \frac{1}{\lambda_{0}} u .
$$

$$
\frac{1}{\lambda_{0}}\left[u-\delta^{*} \frac{\lambda_{0}}{\lambda} v\right] \in K,
$$


and the maximality of $\delta^{*}$ implies $\lambda_{0} \leq \lambda$. Similarly, there exists a maximal $\gamma^{*}>0$ such that

$$
v-\gamma^{*} u \in K \quad \text { and } \quad \frac{1}{\lambda}\left[v-\gamma^{*} \frac{\lambda}{\lambda_{0}} u\right] \in K
$$

hence $\lambda \leq \lambda_{0}$. Thus $\lambda=\lambda_{0}$ and the proof is complete.

The following corollary of Theorem 2.7 will be important in the sequel:

Corollary 2.8. Let $A: E \rightarrow K$ such that $\left.A\right|_{K}: K \rightarrow K$ is a completely continuous, monotone, homogeneous, and strong operator. Furthermore, suppose $\mathcal{N}(A)=\{0\}$. Then $A$ has the unique positive eigenvalue $1 / \lambda_{0}$.

Proof. The existence of $u \in K \backslash\{0\}$ and $\lambda_{0}>0$ such that

$$
u=\lambda_{0} A(u)
$$

follows from property (1) of Theorem 2.7. It follows from property (2) of Theorem 2.7, that $\lambda_{0}$ is unique since $A(E) \subset K$.

Since the operator $A$ is positively homogeneous and there exists a nontrivial solution $u \in K$ to (2.13), the function $\delta u$ will also solve (2.13) for all $\delta>0$. Thus, there exists a line of nontrivial solutions to (2.13) bifurcating from the trivial branch at $\lambda=\lambda_{0}$. A complete generalization of the Krein-Rutman theorem must also demonstrate the simplicity of the principal eigenvalue. Although this step remains to be proven for general strong operators, it is true in the case $A$ is linear:

Corollary 2.9. Let $A: E \rightarrow E$ be a linear, compact, and strong operator and let $u \in K$ satisfy $u=\lambda_{0} A(u)$. If $v \in K \backslash\{0\}$ such that $v=\lambda_{0} A(v)$, then $v=\theta u$ for some $\theta>0$.

Proof. As $A$ is strong and $u, v \in \operatorname{Im}(A) \cap K \backslash\{0\}$, there exists $\delta^{*}>0$, maximal, such that $u-\delta^{*} v \in K$. Furthermore, by linearity of $A$, we have $u-\delta^{*} v=A\left(\lambda_{0}\left(u-\delta^{*} v\right)\right)$. Therefore, if $u-\delta^{*} v \neq 0$, then we may again appeal to the strength of $A$ to conclude there exists $\xi>0$ such that $\left(u-\delta^{*} v\right)-\xi v=$ $\left(u-\left(\delta^{*}+\xi\right) v \in K\right.$, contradicting the maximality of $\delta^{*}$. Therefore $u=\theta v$ for $\theta=\delta^{*}$.

In summary, in this section we established a partial generalization of the Krein-Rutman Theorem, for nonlinear operators which satisfy the "linear-like" properties of homogeneity and monotonicity. The proofs are quite similar to those used for linear operators (e.g. [21], [40]) where linearity conditions are replaced by corresponding monotonicity and homogeneity properties. A complete generalization must also prove simplicity of the eigenvalue. Although this step will be readily established in the applications considered here, it is unclear at this time how to prove this step for a general nonlinear strong operator $A$. 


\section{Principal eigenvalue for $k$-Hessian operator}

Let us now turn to the question of existence of a principal eigenvalue for the $k$-Hessian operator. That is, we shall consider the eigenvalue problem

$$
\begin{cases}S_{k}\left(D^{2} u\right)=|\lambda u|^{k} & x \in \Omega, \\ u=0 & x \in \partial \Omega,\end{cases}
$$

in the class of $k$-convex functions.

One is naturally led to Krein-Rutman type considerations for this problem, in that, any $k$-convex solution to (3.1) is necessarily subharmonic, hence lies in the cone of nonpositive functions in $C(\bar{\Omega})$. Thus, a nontrivial solution to (3.1) will correspond to a positive solution (in the sense of the partial order) to the corresponding eigenvalue problem.

Let $E$ denote the Banach space $C(\bar{\Omega})$ of continuous functions on $\bar{\Omega}$, with the supremum norm $\|\cdot\|=\|\cdot\|_{\infty}$. Consider the following two cones in $E$ :

$$
K=\{u \in E: u(x) \leq 0 \text { for all } x \in \Omega\}
$$

and

$$
K^{+}=\left\{u \in E: u \text { is subharmonic and }\left.u\right|_{\partial \Omega}=0\right\} .
$$

Notice $K^{+} \subset K$, and if $u \in K^{+} \backslash\{0\}$ then $u<0$ in $\Omega$. Applying Definition 2.1 to the cone $K$ we see that

$$
u \leq v \text { in } K \text { if and only if } v(x) \leq u(x) \text { for all } x \in \Omega
$$

Notice that the ordering given by (3.2) is contravariant with respect to the "usual" pointwise ordering. To avoid confusion, we shall always include the explicit dependence on $x$ when considering pointwise relations.

3.1. The solution operator $T_{k}$. In this section we establish the complete continuity of the solution operator for the $k$-Hessian operator in the elliptic case. Let $\Omega$ be a uniformly $(k-1)$-convex domain and consider the equation

$$
\begin{cases}S_{k}\left(D^{2} u\right)=|f| & x \in \Omega, \\ u=0 & x \in \partial \Omega .\end{cases}
$$

Definition (solution operator $T_{k}$ ). Let $f \in E$. For $k=1, \ldots, n$, define the solution operator $T_{k}(f)$ by $T_{k}(f)=u$, where $u \in \Phi^{k}(\Omega) \cap C(\bar{\Omega})$ is the unique $k$-convex admissible solution to (3.3).

It follows from the recent results of Trudinger [43, Theorem 1.1] that $T_{k}$ is well defined and $T_{k}(f) \in C^{0,1}(\bar{\Omega})$. Furthermore, $\operatorname{Im}\left(T_{k}\right) \subset K$. Thus $T_{k}$ is a positive operator. 
Proposition 3.2. For each $k \in\{1, \ldots, n\}$, the operator $T_{k}$ is completely continuous.

Proof. Compactness of $T_{k}$. Let $\left\{f_{m}\right\}$ form a bounded sequence in $E$, say $\left\|f_{m}\right\| \leq M$, and consider the set $\left\{v_{m}\right\}=\left\{T_{k}\left(f_{m}\right)\right\}$. Let $w \in \Phi^{k}(\Omega) \cap C^{\infty}(\Omega)$ denote the unique $k$-convex solution to the equation $w=T_{k}(M)$. By Theorem 1.10, one sees that the inequality $w(x) \leq v_{m}(x)$ holds for all $x \in \Omega$, and for all $m$. Therefore the sequence $\left\{v_{m}\right\}$ also forms a uniformly bounded sequence in $C(\bar{\Omega})$. For any $\Omega^{\prime} \subset \subset \Omega$, the Lipschitz continuity of the functions $v_{m}$ implies that the sequence $\left\{v_{m}\right\}$ forms an equicontinuous family of functions in $C\left(\overline{\Omega^{\prime}}\right)$. Therefore, by the Ascoli-Arzelà theorem, the functions $v_{m}$ converges uniformly on compacta. By using an exhaustion of the domain $\Omega$ we may construct a subsequence of $\left\{v_{m}\right\}$ and a continuous function $v$ such that $v_{m} \rightarrow v$ for all $x \in \Omega$. The presence of the barrier function $w$ implies the limiting function $v$ also satisfies $\left.v\right|_{\partial \Omega}=0$, in which case the sequence $v_{m} \rightarrow v$ uniformly. This completes the proof.

Continuity of $T_{k}$. Let $f_{m} \rightarrow f$ in $E$. In particular, the sequence $\left\{f_{m}\right\}$ is uniformly bounded, say $\left\|f_{m}\right\| \leq M$, for all $m \in \mathbb{N}$. By an identical argument to that above in the proof of compactness, we may find a function $v \in C(\bar{\Omega})$ such that the sequence $v_{m} \rightarrow v$ uniformly. From the characterization of Lemma 1.3, it is clear that $v \in \Phi^{k}(\Omega)$. Therefore, by Theorem 1.8, the associated measures converge weakly; i.e.,

$$
\mu_{k}\left[v_{m}\right] \rightarrow \mu_{k}[v] .
$$

On the other hand, since $\Omega$ is bounded and $\left|f_{m}(x)\right| \leq M$, we see that

$$
\int_{e}\left|f_{m}\right| \rightarrow \int_{e}|f|
$$

Combined with (3.4), this fact implies

$$
\int_{e}|f|=\mu_{k}[v](e)
$$

for all Borel subsets $e \subset \Omega$, in which case $v$ is the unique $k$-convex solution to the equation

$$
\begin{cases}S_{k}\left(D^{2} v\right)=|f| & x \in \Omega, \\ v=0 & x \in \partial \Omega .\end{cases}
$$

Therefore, $T_{k}(f)=v$, which implies the operator $T_{k}$ is continuous.

3.2. The operator $A_{k}$. In connection with (3.1), it will prove convenient to introduce the homogenized operator $A_{k}$ : 
Definition 3.3. Define $A_{k}: E \rightarrow K$ by $A(u)=z$, where $z$ is the unique $k$-convex admissible solution to the equation

$$
\begin{cases}S_{k}\left(D^{2} z\right)=|u|^{k} & x \in \Omega \\ z=0 & x \in \partial \Omega\end{cases}
$$

Notice that if $\lambda>0$ and $u \in K \backslash\{0\}$ such that $u=\lambda A_{k}(u)$, then $(\lambda, u)$ solves (3.1). The operator $A_{k}$ enjoys many nice properties:

1. $A_{k}: K \rightarrow K$ is completely continuous.

Proof. This follows as $A_{k}$ is the composition of the bounded continuous function $u \mapsto|u|^{k}$ with the completely continuous operator $T_{k}$.

2. $\mathcal{N}\left(A_{k}\right)=\{0\}$.

3. $A_{k}$ is homogeneous of degree 1 .

4. $A_{k}$ is monotone.

Proof. Let $u \leq v$ in $E$. Then $v(x) \leq u(x) \leq 0$ for all $x \in \Omega$, hence $|v(x)|^{k} \geq$ $|u(x)|^{k} \geq 0$ for all $x \in \Omega$. By Theorem 1.10, we have $A_{k}(v)(x) \leq A_{k}(u)(x)$ for all $x \in \Omega$, hence $A_{k}(u) \leq A_{k}(v)$ in $E$.

5. Let $u \in \operatorname{Im}\left(A_{k}\right) \backslash\{0\}$. Then $u<0$ in $\Omega$.

Proof. That $u \leq 0$ follows from the comparison principle, Theorem 1.10. The strict inequality follows from the fact that $k$-convex functions are subharmonic and $u$ is not identically zero.

6. Let $u \in \operatorname{Im}\left(A_{k}\right) \backslash\{0\}$. Then for each point $x_{0} \in \partial \Omega$ the exterior normal derivative satisfies $u_{\nu}\left(x_{0}\right)>0$.

Proof. The proof follows from the Hopf lemma [15, Lemma 3.4] as $u$ is subharmonic and $u(x)<0$ for $x \in \Omega$.

Since $u \in \operatorname{Im}\left(A_{k}\right) \backslash\{0\}$ implies $u<0$ in $\Omega$, any nontrivial eigenfunction of $A_{k}$ will lie in the wedge of strictly negative functions, in which case the right-hand side of (3.1) will be strictly positive in $\Omega$ and regularity results of [15] imply $u \in C^{2}(\Omega) \cap C^{1,1}(\bar{\Omega})$.

The properties of $A_{k}$ enumerated above allow a direct application of Corollary 2.8 to establish the following theorem:

Theorem 3.4 (principal eigenvalue for $A_{k}$ ). For each $k \in\{1, \ldots, n\}$, the operator $A_{k}$ has the unique positive eigenvalue $1 / \lambda_{0}$.

Note that by construction, any eigenfunction for $A_{k}$ must lie in the cone $K$, thus $1 / \lambda_{0}$ is the unique eigenvalue associated with a $k$-convex solution. Furthermore, we also have the simplicity and monotonicity of $\lambda_{0}$ : 
THEOREM 3.5 (simplicity and monotonicity of $\lambda_{0}$ ). If $u_{0}$ is an eigenfunction associated with $\lambda_{0}$, then the following statements are true:

(1) If $v \in K \backslash\{0\}$ also satisfies (3.1), then $v=\theta u_{0}$ for some $\theta>0$.

(2) If $\Omega^{\prime} \subset \subset \Omega$, both uniformly ( $\left.k-1\right)$-convex, then the eigenvalue associated with $\Omega^{\prime}$ is strictly greater than the eigenvalue associated with $\Omega$.

Proof. Let $\delta^{*}>0$ be maximal such that $u_{0}-\delta^{*} v \leq 0$ for all $\Omega$. If $u_{0}-\delta^{*} v \equiv$ 0 , then we are done. Let $w=\delta^{*} v$ and consider the linear second-order operator $L_{k}$ defined by

$$
L_{k}=\sum_{i, j=1}^{n} F_{i j}\left(D^{2} w\right) D_{i j}
$$

where $F_{k}=S_{k}^{1 / k}$ and $F_{i j}=\partial F_{k} / \partial r_{i j}$ as discussed in Section 1.2. As $w$ is a $k$-convex solution to (3.1), we may apply Lemma 1.7 to conclude

$$
\begin{aligned}
L_{k}\left(u_{0}-w\right) & \geq F_{k}\left(D^{2} u_{0}\right)-F_{k}\left(D^{2} w\right)=-\lambda_{0} u_{0}-\left(-\lambda_{0} w\right) \\
& =-\lambda_{0}\left(u_{0}-\delta^{*} v\right) \geq 0
\end{aligned}
$$

Thus we see that the function $u_{0}-w$ satisfies

$$
\begin{cases}L_{k}\left(u_{0}-w\right) \geq 0 & \text { in } \Omega, \\ u_{0}-w=0 & \text { on } \partial \Omega .\end{cases}
$$

Therefore, by Hopf's Lemma [15, Lemma 3.4], the outer normal derivative of $u_{0}-w$ satisfies $\left(u_{0}-w\right)_{\nu}>0$ for all $x \in \partial \Omega$. This condition implies, for $\varepsilon>0$ sufficiently small, that $\left(u_{0}-w\right)-\varepsilon v \leq 0$ for all $x \in \Omega$, or $u_{0}-\left(\delta^{*}+\varepsilon\right) v \in K$, contradicting the maximality of $\delta^{*}$. Thus $v=\theta u_{0}$ where $\theta=1 / \delta^{*}>0$.

Next we prove the monotonicity of domain property. Let $\left(\lambda_{0}, u\right),(\lambda, v)$ denote nontrivial eigenpairs for the domains $\Omega$ and $\Omega^{\prime}$ respectively. By scaling, we may assume $u<v$ in $\Omega^{\prime}$. Assume that $\lambda \leq \lambda_{0}$, say $\lambda=\lambda_{0}-\varepsilon$, where $0 \leq \varepsilon<\lambda_{0}$. Let $\delta^{*}>0$ be maximal such that $u-\delta^{*} v \leq 0$ in $\Omega^{\prime}$. Clearly there exists $x_{0} \in \Omega^{\prime}$ such that $\left(u-\delta^{*} v\right)\left(x_{0}\right)=0$; i.e., $u-\delta^{*} v$ achieves an interior maximum. Let $L_{k}$ represent the elliptic operator defined by (3.6), with $w=\delta^{*} v$. Calculating as before, we obtain

$$
\begin{aligned}
L_{k}\left(u-\delta^{*} v\right) & \geq-\lambda_{0} u-\left(\lambda_{0}-\varepsilon\right) \delta^{*} v \\
& =-\lambda_{0}\left(u-\left(1-\varepsilon / \lambda_{0}\right) \delta^{*} v\right)=-\lambda_{0}(u-\gamma v),
\end{aligned}
$$

where $0<\gamma \leq \delta^{*}$. Therefore $L_{k}\left(u-\delta^{*} v\right) \geq 0$, in which case, by the maximum principle, $u-\delta^{*} v \equiv 0$ in $\Omega^{\prime}$. Thus $\left.u\right|_{\partial \Omega^{\prime}}=0$; hence, since $u$ is subharmonic, $u \equiv 0$ in $\Omega$, a contradiction. Therefore $\lambda>\lambda_{0}$. 
Given these properties of $\lambda_{0}$, it is natural to call $\lambda_{0}$ the principal eigenvalue for the $k$-Hessian operator on the domain $\Omega$. The existence of a principal eigenvalue for the $k$-Hessian operator $(1<k<n)$ was first established by Wang [49], using techniques similar to those first used by Lions [29] in the case $k=n$. A related nonlinear eigenvalue result is considered in [9]. In particular, in [9], the authors (among other things) establish the existence of a principal eigenvalue for a large class of ordinary differential operators, which includes the radial case of the $p$-Laplacian and the $k$-Hessian.

\section{Global bifurcation}

In this section we consider the Dirichlet problem

$$
\begin{cases}S_{k}\left(D^{2} u\right)=\lambda\left(|u|^{k}+g(u)\right) & x \in \Omega, \\ u=0 & x \in \partial \Omega,\end{cases}
$$

where the nonhomogeneous term $g: \mathbb{R} \rightarrow[0, \infty)$ satisfies some reasonable properties. We seek values of the parameter $\lambda$ such that (4.1) has nontrivial solutions. Note that the case $g=0$ was considered in Section 3, where it was established that there exists a unique positive value of $\lambda$ such that (4.1) has nontrivial solutions. Thus, we shall assume throughout this section that $g$ is not identically zero.

The semilinear problem associated with (4.1) when $k=1$ (Laplacian) has been studied extensively. For instance, in [35], [34], [41] (and references therein), the authors consider the Dirichlet problem

$$
\begin{cases}L u=\lambda(u+g(u)) & x \in \Omega, \\ u=0 & x \in \partial \Omega,\end{cases}
$$

for $L$ a uniformly elliptic linear second-order differential operator, and obtain results similar in nature to those found here. Many of the techniques employed here are motivated by work in these papers.

Let us briefly recall some results from the theory of global bifurcations which will be essential for our analysis of global bifurcation problems associated with $k$-Hessian operators. By continuum, we shall mean a closed connected set.

Theorem 4.1 (Continuum property, [40]). Let $\mathcal{O}$ be an open bounded subset of $E$ and assume that $F: \mathbb{R} \times E \rightarrow E$ is completely continuous. Furthermore, assume that for $\lambda=\lambda_{0}$ we have $d\left(\mathrm{id}-F\left(\lambda_{0}, \cdot\right), \mathcal{O}, 0\right) \neq 0$. Let

$$
\mathcal{S}^{+}=\left\{(\lambda, u) \in\left[\lambda_{0}, \infty\right) \times E: u=F(\lambda, u)\right\}
$$

and

$$
\mathcal{S}^{-}=\left\{(\lambda, u) \in\left(-\infty, \lambda_{0}\right] \times E: u=F(\lambda, u)\right\} .
$$


Then there exist maximal continua $\mathcal{C}^{+} \subset \mathcal{S}^{+}$and $\mathcal{C}^{-} \subset \mathcal{S}^{-}$, and for both $C=\mathcal{C}^{-}$ and $C=\mathcal{C}^{+}$, the following statements are valid:

(1) $C \cap\left\{\lambda_{0}\right\} \times \mathcal{O} \neq \emptyset$.

(2) Either $C$ is unbounded or else $C \cap\left\{\lambda_{0}\right\} \times E \backslash \overline{\mathcal{O}} \neq \emptyset$.

TheOREM 4.2 (global bifurcation, [24]). Let $F: \mathbb{R} \times E \rightarrow E$ be completely continuous such that $F(\lambda, 0)=0$, for all $\lambda \in \mathbb{R}$. Suppose there exist constants $a, b \in \mathbb{R}$, with $a<b$, such that $(a, 0)$ and $(b, 0)$ are not bifurcation points for the equation

$$
u-F(\lambda, u)=0
$$

Furthermore, assume that

$$
d\left(\mathrm{id}-F(a, \cdot), B_{r}(0), 0\right) \neq d\left(\mathrm{id}-F(b, \cdot), B_{r}(0), 0\right),
$$

where $B_{r}(0)=\{u \in E:\|u\|<r\}$ is an isolating neighbourhood of the trivial solution for both constants $a$ and $b$. Let

$$
\mathcal{S}=\overline{\{(\lambda, u):(\lambda, u) \text { is a solution of }(4.2) \text { with } u \neq 0\}} \cup([a, b] \times\{0\}),
$$

and let $\mathcal{C}$ be the component of $\mathcal{S}$ containing $[a, b] \times\{0\}$. Then either

(1) $\mathcal{C}$ is unbounded in $\mathbb{R} \times E$, or

(2) $\mathcal{C} \cap[(\mathbb{R} \backslash[a, b]) \times\{0\}] \neq \emptyset$.

TheOrem 4.3 (global asymptotic bifurcation, [24]). Let $F: \mathbb{R} \times E \rightarrow E$ be completely continuous and suppose there exist constants $a, b \in \mathbb{R}$, with $a<b$, such that solutions of (4.2) are a priori bounded in $E$ for $\lambda=a$ and $\lambda=b$; i.e., there exists a constant $M>0$ such that

$$
F(a, u) \neq u \neq F(b, u),
$$

for all $u \in E$ with $\|u\| \geq M$. Furthermore, assume that

$$
d\left(\mathrm{id}-F(a, \cdot), B_{R}(0), 0\right) \neq d\left(\mathrm{id}-F(b, \cdot), B_{R}(0), 0\right),
$$

for $R>M$. Then there exists at least one continuum $\mathcal{C}$ of solutions to (4.2) that is unbounded in $[a, b] \times E$ and either

(1) $\mathcal{C}$ is unbounded in the $\lambda$ direction, or else,

(2) there exists an interval $[c, d]$ such that $(a, b) \cap(c, d)=\emptyset$, and $\mathcal{C}$ bifurcates from infinity in $[c, d] \times E$.

Theorem 4.2 equates a degree change to the existence of a continuum of nontrivial solutions which bifurcates from the trivial branch and is either unbounded in the product space or meets another bifurcation point outside the given interval $[a, b]$. Theorem 4.3 equates a priori bounds together with a degree change to the 
existence of a continuum which bifurcates at infinity in the interval $[a, b]$ and is either unbounded in the $\lambda$ direction or has another asymptotic bifurcation value outside the interval $[a, b]$. Note that Theorem 4.3 does not require the trivial branch of solutions and there may be no bifurcation for (4.2) in the classical sense.

This remainder of this section is organized into three parts. The first subsection contains an elementary degree calculation which we shall need in the sections that follow. The behavior of the solution set to (4.1) naturally depends on the growth conditions assumed on $g$, which we have divided into the two remaining subsections. Recall that $E$ is the Banach space of continuous functions defined on $\bar{\Omega}$, where $\Omega$ is any bounded strictly $(k-1)$-convex domain in $\mathbb{R}^{n}$.

4.1. An elementary degree calculation. In this section we perform an elementary degree calculation for the operator $A_{k}$ defined in Section 3.2.

LEMmA 4.4. For any $R>0$, there exists $\lambda_{a} \geq 0$ and $\lambda_{b}>\lambda_{0}$ such that

$$
d\left(\mathrm{id}-\lambda_{a} A_{k}(\cdot), B_{R}(0), 0\right) \neq d\left(\mathrm{id}-\lambda_{b} A_{k}(\cdot), B_{R}(0), 0\right)
$$

Proof. Fix $R>0$. One may simply choose $\lambda_{a}=0$ and conclude

$$
d\left(\mathrm{id}-\lambda_{a} A_{k}(\cdot), B_{R}(0), 0\right)=d\left(\mathrm{id}, B_{R}(0), 0\right)=1 .
$$

However, in fact, (4.3) holds for all $\lambda_{a}>0$ sufficiently small. For if not, then there exist sequences $\left\{\lambda_{m}\right\}$ and $\left\{u_{m}\right\}$ satisfying $\lambda_{m} \rightarrow 0,\left\|u_{m}\right\|=R$, and

$$
u_{m}=\lambda_{m} A_{k}\left(u_{m}\right)
$$

The complete continuity of $A_{k}$ applied to (4.4) implies there exists a solution $u$, with $\|u\|=R$, such that $u_{m} \rightarrow u$. However (4.4) combined with the fact that $\lambda_{m} \rightarrow 0$ implies $\|u\|=0$, a contradiction. Therefore we may choose any positive constant $\lambda_{a}$ such that $\lambda_{a}<\lambda_{0}$ and (4.3) holds.

Next we show that there exists a constant $\lambda_{b}>\lambda_{0}$ such that

$$
d\left(\mathrm{id}-\lambda_{b} A_{k}(\cdot), B_{R}(0), 0\right)=0
$$

By the strength of $A_{k}$, we may find $w \in K \backslash\{0\}$ such that $w \leq \beta A_{k}(w)$ for some $\beta>0$. Furthermore, by Theorem 2.3, $\lambda_{0} \leq \beta$. Choose $\lambda_{b}>\beta$. By the continuity of the Leray-Schauder degree we have

$$
d\left(\mathrm{id}-\lambda_{b} A_{k}(\cdot), B_{R}(0), 0\right)=d\left(\mathrm{id}-\lambda_{b} A_{k}(\cdot+\varepsilon w), B_{R}(0), 0\right),
$$

for all $\varepsilon>0$, sufficiently small. Since $\lambda_{b}>\beta$, we may apply Lemma 2.4 to conclude the equation

$$
u=\lambda_{b} A_{k}(u+\varepsilon w)
$$


has no solutions in E. Therefore,

$$
d\left(\mathrm{id}-\lambda_{b} A_{k}(\cdot+\varepsilon w), B_{R}(0), 0\right)=d\left(\mathrm{id}-\lambda_{b} A_{k}(\cdot), B_{R}(0), 0\right)=0 .
$$

This completes the degree calculation.

4.2. The $k$-superlinear case. With (4.1) in mind, consider the perturbed Dirichlet problem

$$
\begin{cases}S_{k}\left(D^{2} z\right)=|u|^{k}+g(u) & x \in \Omega, \\ z=0 & x \in \partial \Omega,\end{cases}
$$

where the perturbation term $g$ satisfies the conditions

(1) $g: \mathbb{R} \rightarrow[0, \infty)$ is continuous,

(2) $g=o\left(|u|^{k}\right)$ as $|u| \rightarrow 0$.

For instance, we may consider $g(u)=|\delta u|^{p}$ for $\delta \in \mathbb{R}$ and $p>k$.

Condition (2) is often referred to as a superlinear growth condition near the origin. To be precise, we shall call it $k$-superlinear growth, to emphasize the growth is superlinear near the origin relative to the power $|u|^{k}$.

Let $B_{k}=T_{k} \circ N_{k}$ denote the solution operator to (4.5), where $T_{k}$ is the solution operator introduced in Section 3, and $N_{k}$ is the Nemytskiu operator defined by $N_{k}(u)=|u|^{k}+g(u)$. Note that, since $g$ is nonnegative, $N_{k}(E) \subseteq$ $E^{+}=\{u \in E: u(x) \geq 0$ for all $x \in \Omega\}$.

As $B_{k}$ is the composition of a bounded, continuous operator with a completely continuous operator, $B_{k}$ is also completely continuous. Define $j_{k}$ : $\mathbb{R} \times E \rightarrow E$ by

$$
j_{k}(\lambda, w)=w-|\lambda| B_{k}(w)
$$

and consider the equation

$$
j_{k}(\lambda, u)=u-|\lambda| B_{k}(u)=0 .
$$

Notice that $j_{k}(\lambda, u)=0$ implies $u \in K$, and $u$ is an admissible solution to the Dirichlet problem

$$
\begin{cases}S_{k}\left(D^{2} u\right)=|\lambda u|^{k}+|\lambda|^{k} g(u) & x \in \Omega, \\ u=0 & x \in \partial \Omega .\end{cases}
$$

Since $B_{k}(0)=T_{k}\left(N_{k}(0)\right)=0$, the equation $j_{k}(\lambda, 0)=0$ has the trivial solution for all values of $\lambda \in \mathbb{R}$. We are interested in finding nontrivial branches of solutions to (4.7) which bifurcate from this trivial branch.

We shall establish the existence of a global branch of nontrivial solutions to (4.7) which bifurcates from the trivial branch at $\lambda=\lambda_{0}$, where $\lambda_{0}$ is the principal eigenvalue for the $k$-Hessian operator on the domain $\Omega$. Namely, we prove the following theorem: 
THEOREM 4.5 (global bifurcation for (4.6)). The point $(\mu, 0)$ is a bifurcation point for (4.6) if and only if $|\mu|=\lambda_{0}$. Furthermore, there exists an unbounded continuum $\mathcal{C}^{+}$(resp. $\mathcal{C}^{-}$) of nontrivial solutions to (4.6), which bifurcates from $\left(\lambda_{0}, 0\right)\left(\left(-\lambda_{0}, 0\right)\right)$ and lies in the strip $\left\{(\lambda, u): 0 \leq \lambda \leq \lambda_{0}\right\}$ (resp. $\left\{(\lambda, u):-\lambda_{0} \leq\right.$ $\lambda \leq 0\})$.

Proof. Necessity. Suppose $(\mu, 0)$ is a bifurcation point for (4.6). Then there exists a sequence $\left(\lambda_{m}, u_{m}\right) \rightarrow(\mu, 0)$ such that $\left\|u_{m}\right\| \neq 0$ for all $k$, and $j_{k}\left(\lambda_{m}, u_{m}\right)=0$; i.e., the components of $\left(\lambda_{m}, u_{m}\right)$ solve the equation

$$
\begin{cases}S_{k}\left(D^{2} u_{m}\right)=\left|\lambda_{m} u_{m}\right|^{k}+\left|\lambda_{m}\right|^{k} g\left(u_{m}\right) & x \in \Omega, \\ u_{m}=0 & x \in \partial \Omega .\end{cases}
$$

Let $v_{m}=u_{m} /\left\|u_{m}\right\|$. Then $\left\|v_{m}\right\|=1$ and by dividing (4.8) by $\left\|u_{m}\right\|^{k}$ we see that $v_{m}$ is the unique admissible solution to

$$
v_{m}=T_{k}\left(f_{m}\left(v_{m}\right)\right)
$$

where the operators $f_{m}: E \rightarrow E^{+}$are defined by

$$
f_{m}(w)=\left|\lambda_{m} w\right|^{k}+\left|\lambda_{m}\right|^{k} \frac{g\left(u_{m}\right)}{\left\|u_{m}\right\|^{k}} .
$$

We next show that the set of functions $\left\{f_{m}\left(v_{m}\right)\right\}$ is bounded in $E$, so that we may apply the complete continuity of the operator $T_{k}$ to (4.9). To see this, first let us introduce the function $\phi_{k}: \mathbb{R} \rightarrow[0, \infty)$ defined by

$$
\phi_{k}(\xi)= \begin{cases}\frac{g(\xi)}{|\xi|^{k}} & \text { if } \xi \neq 0, \\ 0 & \text { otherwise. }\end{cases}
$$

The conditions imposed on $g$ above imply $\phi_{k} \circ u: \bar{\Omega} \rightarrow[0, \infty)$ is a bounded continuous function for any $u \in E$. Using $\phi_{k}$, we may express (4.10) in the following equivalent form (with $w=v_{m}$ )

$$
f_{m}\left(v_{m}\right)=\left|\lambda_{m} v_{m}\right|^{k}\left(1+\phi_{k}\left(u_{m}\right)\right) .
$$

The condition $\left\|u_{m}\right\| \rightarrow 0$ implies the functions $u_{m} \rightarrow 0$ uniformly on $\bar{\Omega}$; hence the functions $\phi_{k}\left(u_{m}\right)$ also tend to 0 uniformly on $\bar{\Omega}$, and in particular, are uniformly bounded. Therefore, since $\left\{\phi_{k}\left(u_{m}\right)\right\},\left\{\lambda_{m}\right\}$, and $\left\{v_{m}\right\}$ are all bounded, we may conclude that the set $\left\{f_{m}\left(v_{m}\right)\right\}$ is bounded in $E$. Hence, we may apply the complete continuity of $T_{k}$ to (4.9) to obtain a convergent subsequence of $\left\{v_{m}\right\}$, with $v_{m_{j}} \rightarrow v \neq 0$, as $\left\|v_{m}\right\|=1$ for all $m$.

Armed with this knowledge, we may reexamine (4.12) to conclude that the functions $f_{m}\left(v_{m}\right) \rightarrow|\mu v|^{k}$. Coupled with (4.9), this implies $v=T_{k}\left(|\mu v|^{k}\right)$; i.e., 
$v$ satisfies the equation

$$
\begin{cases}S_{k}\left(D^{2} v\right)=|\mu v|^{k} & x \in \Omega, \\ v=0 & x \in \partial \Omega,\end{cases}
$$

or $v$ is an eigenfunction associated to the eigenvalue $\mu$ for the $k$-Hessian operator. Therefore, by Theorem $3.4,|\mu|=\lambda_{0}$. Thus $\pm \lambda_{0}$ are the only possible bifurcation points for (4.6).

Sufficiency. We shall prove that $\lambda_{0}$ corresponds to a bifurcation point for (4.6) by an application of Theorem 4.2. Clearly the operator $|\lambda| B_{k}: \mathbb{R} \times E \rightarrow E$ is completely continuous, as the operator $B_{k}$ is. For simplicity, we shall assume $\lambda \geq 0$, the case $\lambda \leq 0$ is handled in a similar fashion. To apply Theorem 4.2 , we must find constants $\lambda_{a}$ and $\lambda_{b}$, with $\lambda_{a}<\lambda_{0}<\lambda_{b}$, such that $u=0$ is an isolated solution of (4.6) for $\lambda \in\left\{\lambda_{a}, \lambda_{b}\right\}$ and

$$
d\left(\mathrm{id}-\lambda_{a} B_{k}(\cdot), B_{R}(0), 0\right) \neq d\left(\mathrm{id}-\lambda_{b} B_{k}(\cdot), B_{R}(0), 0\right) .
$$

Here $B_{R}(0)$ should be an isolating neighbourhood of the trivial solution for both $\lambda_{a}$ and $\lambda_{b}$. Equivalently,

$$
d\left(\mathrm{id}-T_{k}\left(\lambda_{a}^{k}|\cdot|^{k}+\lambda_{a}^{k} g(\cdot)\right), B_{R}(0), 0\right) \neq d\left(\mathrm{id}-T_{k}\left(\lambda_{b}^{k}|\cdot|^{k}+\lambda_{b}^{k} g(\cdot)\right), B_{R}(0), 0\right) .
$$

First, notice that for any $\mu \geq 0$, with $\mu \neq \lambda_{0}$, the function $u=0$ is an isolated solution of (4.6), by the proof of necessity given above. Second, for any constant $\mu \geq 0$, with $\mu \neq \lambda_{0}$, and for all $R>0$, sufficiently small, the family of mappings $H_{k}:[0,1] \times \overline{B_{R}(0)} \rightarrow E$ defined by

$$
H_{k}(t, u)=T_{k}\left(\mu^{k}|u|^{k}+t \mu^{k} g(u)\right),
$$

is a degree preserving homotopy between the operators $T_{k}\left(\mu^{k}|\cdot|^{k}+\mu^{k} g(\cdot)\right)$ and $T_{k}\left(\mu^{k}|\cdot|^{k}\right)$. To see this, it suffices to show there exists $\varepsilon>0$, sufficiently small, such that the equation

$$
u-T_{k}\left(\mu^{k}|u|^{k}+t \mu^{k} g(u)\right)=0
$$

has no solutions $u \in E$, with $\|u\|=\varepsilon$, for any $t \in[0,1]$. For if not, then we may construct sequences $\left\{\varepsilon_{m}\right\},\left\{t_{m}\right\} \subset[0,1]$, and $\left\{u_{m}\right\}$ satisfying $\left\|u_{m}\right\|=\varepsilon_{m} \rightarrow 0$, such that

Equivalently,

$$
u_{m}=T_{k}\left(\mu^{k}\left|u_{m}\right|^{k}+t_{m} \mu^{k} g\left(u_{m}\right)\right) \text {. }
$$

$$
S_{k}\left(D^{2} u_{m}\right)=\left|\mu u_{m}\right|^{k}+t_{m} \mu^{k} g\left(u_{m}\right) .
$$

Now we are back in the situation encountered previously, in that we may divide by $\left\|u_{m}\right\|^{k}$ and extract a convergent subsequence of unit vectors, $v_{m} \rightarrow v$, where $v$ satisfies $S_{k}\left(D^{2} v\right)=|\mu v|^{k}$; a contradiction, since $\mu \neq \lambda_{0}$. 
Therefore it is sufficient to find $\lambda_{a}<\lambda_{0}<\lambda_{b}$ such that

$$
d\left(\mathrm{id}-T_{k}\left(\lambda_{a}^{k}|\cdot|^{k}\right), B_{R}(0), 0\right) \neq d\left(\mathrm{id}-T_{k}\left(\lambda_{b}^{k}|\cdot|^{k}\right), B_{R}(0), 0\right),
$$

where $B_{R}(0)$ is a sufficiently small isolating neighbourhood of the trivial solution. Since $T_{k}\left(\lambda^{k}|\cdot|^{k}\right)=\lambda A_{k}(\cdot)$, it suffices to find $\lambda_{a}<\lambda_{0}<\lambda_{b}$ such that

$$
d\left(\mathrm{id}-\lambda_{a} A_{k}(\cdot), B_{R}(0), 0\right) \neq d\left(\mathrm{id}-\lambda_{b} A_{k}(\cdot), B_{R}(0), 0\right) .
$$

However, this is precisely the conclusion of Lemma 4.4, which is valid for any $R>0$. Therefore, if we let $\mathcal{S}$ be defined by

$$
\mathcal{S}=\overline{\{(\lambda, u):(\lambda, u) \text { is a solution of (4.6) with } u \neq 0\}} \cup\left(\left[\lambda_{a}, \lambda_{b}\right] \times\{0\}\right),
$$

then, by Theorem 4.2, there exists a connected component $\mathcal{C}^{+}$of $\mathcal{S}$ containing $\left[\lambda_{a}, \lambda_{b}\right] \times\{0\}$ which is either

(a) unbounded in $\mathbb{R} \times E$, or

(b) $\mathcal{C}^{+} \cap\left[\left(\mathbb{R} \backslash\left[\lambda_{a}, \lambda_{b}\right]\right) \times\{0\}\right] \neq \emptyset$.

Let us examine the possible behavior for the global continuum $\mathcal{C}^{+}$. Since $u=0$ is the unique solution corresponding to $\lambda=0$, the continuum $\mathcal{C}^{+}$cannot cross the $\lambda=0$ axis, other than at the trivial crossing, where $u=0$. Furthermore, since $u=0$ is an isolated solution for (4.6) for all $\lambda \geq 0$, with $\lambda \neq \lambda_{0}$. Alternative (b) above must not hold. Therefore we may conclude $\mathcal{C}^{+}$is unbounded in $\mathbb{R} \times E$.

It remains to show that the continuum lies in the desired strip. Clearly $\lambda \geq 0$. Assume $(\lambda, u)$ solves $j_{k}(\lambda, u)=0$ with $\lambda>\lambda_{0}$ and $u \neq 0$. Let $u_{0}$ be an eigenfunction corresponding to the principal eigenvalue for the $k$-Hessian operator for the domain $\Omega$; i.e., $u_{0}$ satisfies

$$
\begin{cases}S_{k}\left(D^{2} u_{0}\right)=\left|\lambda_{0} u_{0}\right|^{k} & x \in \Omega, \\ u_{0}=0 & x \in \partial \Omega .\end{cases}
$$

By scaling if necessary, we may assume $u(x)<u_{0}(x)$ for all $x \in \Omega$. Let $\delta^{*}>0$ be maximal such that $u-\delta^{*} u_{0} \leq 0$ in $\Omega$. Let $L_{k}$ be the operator defined by (3.6), where $w=\delta^{*} u_{0}$. Employing Lemma 1.7, we compute

$$
\begin{aligned}
L_{k}\left(u-\delta^{*} u_{0}\right) & \geq F_{k}\left(D^{2} u\right)-F_{k}\left(D^{2} w\right) \\
& =\lambda|u|\left[1+\frac{g(u)}{|u|^{k}}\right]^{1 / k}-\lambda_{0}\left|\delta^{*} u_{0}\right| \geq \lambda|u|-\lambda_{0}\left|\delta^{*} u_{0}\right| \geq 0,
\end{aligned}
$$

since $\lambda>\lambda_{0}$ and $0<\left|\delta^{*} u_{0}\right| \leq|u|$ for all $x \in \Omega$. This implies, by the maximum principle, $u=\delta^{*} u_{0}$ for all $x \in \Omega$. Therefore, $S_{k}\left(D^{2} u\right)=S_{k}\left(D^{2} w\right)$, or

$$
\lambda^{k}\left(|u|^{k}+g(u)\right)=\left|\lambda_{0} \delta^{*} u_{0}\right|^{k}=\left|\lambda_{0} u\right|^{k} .
$$

Hence

$$
\lambda^{k}-\lambda_{0}^{k}=-\lambda^{k} \frac{g(u)}{|u|^{k}} \leq 0,
$$


a contradiction, as $\lambda>\lambda_{0}$.

The same reasoning above establishes the existence of a symmetric continuum $\mathcal{C}^{-}$, corresponding to the bifurcation from $-\lambda_{0}$.

We remark that by inequality (4.15) we can infer the following more precise information about the continuum $\mathcal{C}^{+}$:

(1) $\mathcal{C}^{+} \subset\left(0, \lambda_{0}\right] \times E$,

(2) if $(\lambda, u) \in \mathcal{C}^{+}$with $g(u) \neq 0$, then $0<\lambda<\lambda_{0}$.

Therefore we have established the existence of a global continuum $\mathcal{C}^{+}$which is unbounded in the space $\left(0, \lambda_{0}\right] \times E$ and bifurcates from the trivial branch at $\left(\lambda_{0}, 0\right)$, such that the components of an element $(\lambda, u) \in \mathcal{C}^{+}$satisfy (4.1). A possible diagram of $\mathcal{C}^{+}$is sketched in Figure 4.1.

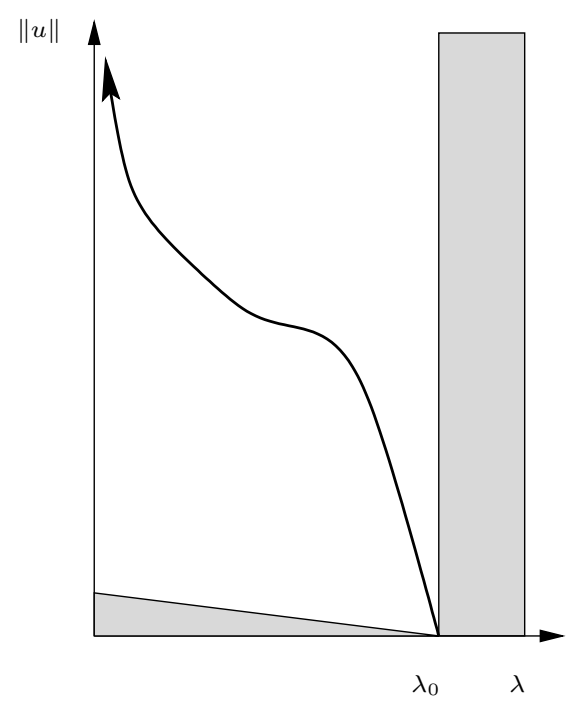

Figure 4.1. Continuum $\mathcal{C}^{+}$of Theorem 4.5

4.3. The $k$-sublinear case - bifurcation at infinity. The bifurcation results above establish the existence of solutions to (4.1) for $g(u)$ higher order than $|u|^{k}$ near 0 . Similar assumptions on $g$ near infinity yield corresponding results. Namely, let us assume the perturbation $g$ is of lower order than $|u|^{k}$ near infinity. More specifically, we will now assume $g: \mathbb{R} \rightarrow[0, \infty)$ satisfies the following conditions:

(1) $g$ is continuous,

(2) $g=o\left(|u|^{k}\right)$ as $|u| \rightarrow \infty$.

Condition (2) above is often referred to as a sublinear growth condition near infinity. In accord with our previous notation, we shall call it a $k$-sublinear growth 
condition on $g$. In this setting we shall also assume $g$ satisfies the additional hypothesis

(3) $g$ is nondecreasing.

For instance, we may consider $g(u)=|\delta u|^{p}$, for $\delta \in \mathbb{R}$, and $0 \leq p<k$. Note that, in general, we no longer require $g(0)=0$. Thus, (4.6) may no longer have the trivial solution for all $\lambda$. For 'large' solutions we recall the following definition:

DEFINITION 4.6 (bifurcation at infinity). The constant $\lambda_{0}$ is a bifurcation value at infinity (or asymptotic bifurcation value) for (4.6) if and only if there exist solutions $\left(\lambda_{m}, u_{m}\right)$ of (4.6) such that $\lambda_{m} \rightarrow \lambda_{0}$ and $\left\|u_{m}\right\| \rightarrow \infty$ as $m \rightarrow \infty$.

For the lower order perturbation we have the following theorem (see Figure 4.2$)$ :

THEOREM 4.7 (global asymptotic bifurcation for (4.6)). Let $g$ satisfy conditions (1), (2), and (3) above. Then $\mu$ is an asymptotic bifurcation value for (4.6) if and only if $|\mu|=\lambda_{0}$. Furthermore, there exists an unbounded continuum of nontrivial solutions to (4.6), which lies in the strip $\left\{(\lambda, u):-\lambda_{0} \leq \lambda \leq \lambda_{0}\right\}$.

Proof. Necessity. Let $\mu$ be an asymptotic bifurcation value for (4.6). Then there exist sequences $\left\{\lambda_{m}\right\}$ and $\left\{u_{m}\right\}$, such that $\lambda_{m} \rightarrow \mu,\left\|u_{m}\right\| \rightarrow \infty$, and the equation

$$
S_{k}\left(D^{2} u_{m}\right)=\left|\lambda_{m} u_{m}\right|^{k}+\left|\lambda_{m}\right|^{k} g\left(u_{m}\right),
$$

is satisfied. Proceeding as before, we obtain unit vectors $v_{m}=u_{m} /\left\|u_{m}\right\|$ satisfying the equation

$$
v_{m}=T_{k}\left(f_{m}\left(v_{m}\right)\right)
$$

where the operators $f_{m}$ are defined by (4.10). By condition (3) on $g$, we have

$$
f_{m}(w) \leq\left|\lambda_{m}\right|^{k}\left[|w|^{k}+\frac{g\left(\left\|u_{m}\right\|\right)}{\left\|u_{m}\right\|^{k}}\right] .
$$

Therefore, by condition (2), the functions $f_{m}\left(v_{m}\right)$ are uniformly bounded in $E$; hence by (4.16), there exists a unit vector $v$ such that $v_{m} \rightarrow v$. Equations (4.16) and (4.17), together with condition (2) on $g$, imply that $v$ solves the equation

$$
\begin{cases}S_{k}\left(D^{2} v\right)=|\mu v|^{k} & x \in \Omega, \\ v=0 & x \in \partial \Omega .\end{cases}
$$

Thus, by Theorem $3.4,|\mu|=\lambda_{0}$.

Sufficiency. We first show that $\lambda_{0}$ is an asymptotic bifurcation values for (4.6). To apply Theorem 4.3, it suffices to find constants $\lambda_{a}, \lambda_{b} \geq 0$ and $M>0$, such that solutions to (4.6) satisfy

$$
T_{k}\left(\left|\lambda_{a} u\right|^{k}+\lambda_{a}^{k} g(u)\right) \neq u \neq T_{k}\left(\left|\lambda_{b} u\right|^{k}+\lambda_{b}^{k} g(u)\right),
$$


for all $u \in E$ with $\|u\| \geq M$, and

$$
\begin{aligned}
d\left(\mathrm{id}-T_{k}\left(\left|\lambda_{a} u\right|^{k}+\lambda_{a}^{k} g(u)\right), B_{R}(0), 0\right) & \\
& \neq d\left(\mathrm{id}-T_{k}\left(\left|\lambda_{b} u\right|^{k}+\lambda_{b}^{k} g(u)\right), B_{R}(0), 0\right),
\end{aligned}
$$

for $R>M$. Condition (4.18) is readily satisfied by choosing any constants $\lambda_{a}$ and $\lambda_{b}$ such that $\lambda_{a} \neq \lambda_{0} \neq \lambda_{b}$, as we have already shown that $\lambda_{0}$ is the only positive asymptotic bifurcation value for (4.6). Furthermore, by using a homotopy argument, dual to the case considered in Theorem 4.5 (now using condition (3) on $g$ ), one sees that (4.19) is equivalent to

$$
d\left(\mathrm{id}-\lambda_{a} A_{k}(\cdot), B_{R}(0), 0\right) \neq d\left(\mathrm{id}-\lambda_{b} A_{k}(\cdot), B_{R}(0), 0\right)
$$

for all $R>0$, sufficiently large. However, this is precisely the conclusion of Lemma 4.4. Therefore, by Theorem 4.3, there exists a continuum $\mathcal{C}^{\infty}$ of solutions to (4.6) that is unbounded in $\left[\lambda_{a}, \lambda_{b}\right] \times E$, and is either

(1) unbounded in the $\lambda$ direction, or else,

(2) there exists an interval $[c, d]$ such that $\left(\lambda_{a}, \lambda_{b}\right) \cap(c, d)=\emptyset$, and $\mathcal{C}^{\infty}$ bifurcates from infinity in $[c, d] \times E$.

However, arguing exactly as in the proof of Theorem 4.5, we may show the continuum lies completely in the strip $\left\{(\lambda, u):-\lambda_{0} \leq \lambda \leq \lambda_{0}\right\}$; hence the continuum is not unbounded in the $\lambda$ direction, and alternative (2) above must hold. We have shown that $\pm \lambda_{0}$ are the only possible asymptotic bifurcation values for (4.6), thus $-\lambda_{0}$ is also an asymptotic bifurcation value for (4.6). Since the continuum may not cross the $\lambda=0$ axis, other than at $(0,0)$, we see that the continuum must pass through the origin. In the case that $g(0)=0$ this would imply that the origin must correspond to a bifurcation value for (4.6) as well. This completes the proof.

As expected, there is a dual nature to the results for the two cases $g=o\left(|u|^{k}\right)$ as $|u| \rightarrow 0$ and $g(u)=o\left(|u|^{k}\right)$ as $|u| \rightarrow \infty$. The reader should compare Figure 4.1 and Figure 4.2 .

\section{Global bifurcation and critical exponents}

In this section we consider the nonlinear $k$-Hessian equation

$$
\begin{cases}S_{k}\left(D^{2} u\right)=g(u) & x \in \Omega, \\ u=0 & x \in \partial \Omega,\end{cases}
$$

on the strictly $(k-1)$-convex domain $\Omega \subset \mathbb{R}^{n}$, for $g: \mathbb{R} \rightarrow[0, \infty)$, continuous. We seek nontrivial solutions to (5.1). The approach we use is to embed (5.1) 


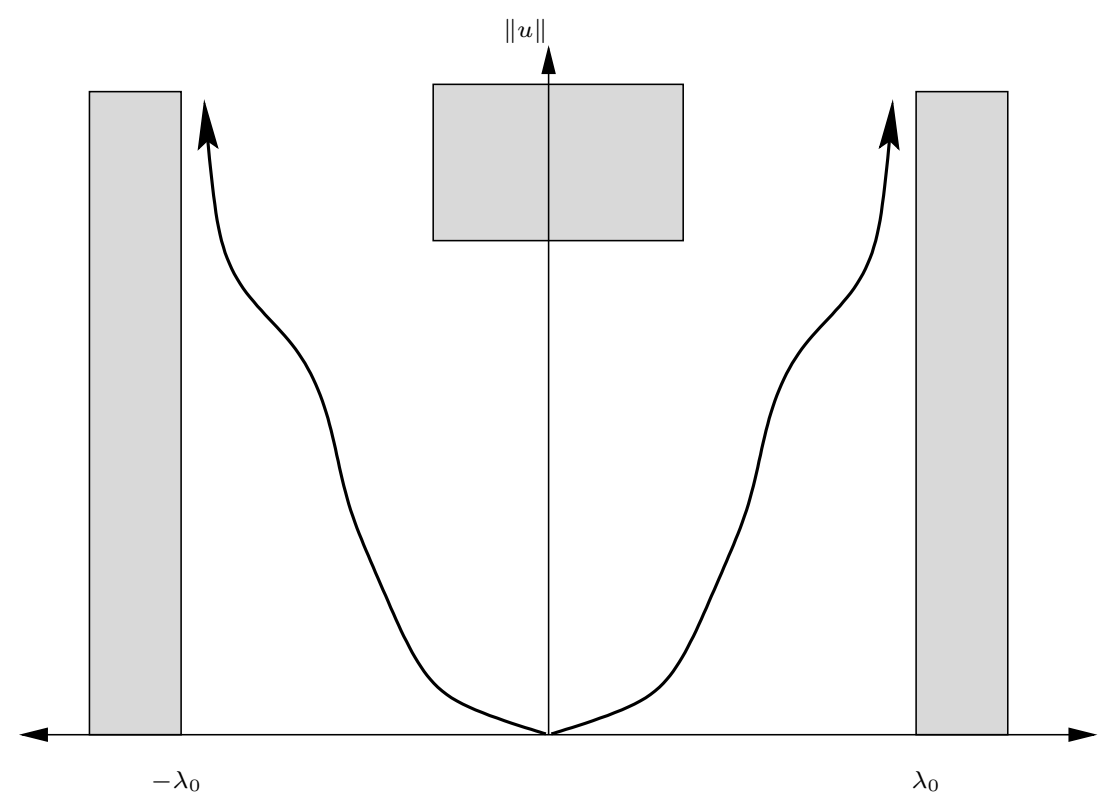

Figure 4.2. Continuum $\mathcal{C}^{\infty}$ of Theorem 4.7

into the one parameter family of equations

$$
\begin{cases}S_{k}\left(D^{2} u\right)=|\lambda u|^{k}+g(u) & x \in \Omega, \\ u=0 & x \in \partial \Omega,\end{cases}
$$

and consider the behavior of global bifurcation continua.

It is in this section that the most striking differences between the $k$-Hessian operators appear. In particular, our approach to (5.1) will depend on the geometry of $k$-convex functions. For instance, we shall see that in the Monge-Ampère case $(k=n)$ there is no critical exponent associated to (5.1). On the other hand, it is well-known that (5.1) has no positive solution in the case $k=1$, when $g(u)=u^{p}$ for $p \geq(n+2) /(n-2), n \geq 3$, and $\Omega$ is starshaped.

We divide the study of (5.1) up into three parts. We begin by examining some general results for (5.1) which hold for each $k \in\{1, \ldots, n\}$. Next, we study (5.2) in the $k$-sublinear case. We will prove that there exists a global continuum of nontrivial solutions to (5.2) which crosses the $\lambda=0$ axis nontrivially. By "crossing the $\lambda=0$ axis nontrivially" we mean the continuum crosses the $\lambda=0$ axis at a point $(0, u)$ with $u \neq 0$. Therefore (5.1) admits a nontrivial solution. Moreover, we will prove that this solution is unique.

The $k$-superlinear case turns out to be a bit more delicate in the general case; hence, we divide the study of this case into two parts. First, we study the MongeAmpère case. Here we shall see how the natural convexity of solutions may be used. For instance, using convexity, we will prove that the global continuum 
of nontrivial solutions to (5.2) must cross the $\lambda=0$ axis nontrivially, thereby exhibiting a nontrivial solution to (5.1). In this manner, we demonstrate that there is no critical exponent for the Monge-Ampère operator. Finally, we return to the general superlinear case of (5.1). Here we discuss some relevant questions in the theory of critical exponents. These sections will illuminate some properties concerning the geometry of $k$-convex functions.

5.1. General results. We begin with two lemmas concerning the norms of solutions to $(5.1)$.

LEMMA 5.1. Let $\left\{v_{m}\right\} \subset C(\bar{\Omega})$ be a collection of $k$-convex solutions to the Dirichlet problem

$$
\begin{cases}S_{k}\left(D^{2} v_{m}\right)=g_{m} & x \in \Omega, \\ v_{m}=0 & x \in \partial \Omega,\end{cases}
$$

where $g_{m}: \Omega \rightarrow \mathbb{R}$ form a collection of nonnegative continuous functions. If $g_{m} \rightarrow \infty$, uniformly on compacta, then $\left\|v_{m}\right\| \rightarrow \infty$.

Proof. Let $\Omega^{\prime} \subset \Omega$ be a strictly $(k-1)$-convex subdomain. Let $\lambda$ be the principal eigenvalue for the $k$-Hessian operator on the domain $\Omega^{\prime}$. Choose $t \in \mathbb{N}$ and let $u$ be an eigenfunction for the domain $\Omega^{\prime}$ with $\|u\|=t$. As $g_{m} \rightarrow \infty$ uniformly on $\Omega^{\prime}$, there exists $m \gg 1$ such that

$$
g_{m} \geq \sup _{\Omega^{\prime}}|\lambda u|^{k} \quad \text { for all } x \in \Omega^{\prime} .
$$

Therefore, by Theorem 1.10, $v_{m}(x) \leq u(x)$ for all $x \in \Omega^{\prime}$. This implies $\left\|v_{m}\right\| \geq$ $\|u\|=t$. As $t$ was arbitrary, the result follows.

Lemma 5.2. Let $\left\{v_{m}\right\} \subset C(\bar{\Omega})$ be a collection of $k$-convex solutions to the Dirichlet problem

$$
\begin{cases}S_{k}\left(D^{2} v_{m}\right)=g_{m} & x \in \Omega, \\ v_{m}=0 & x \in \partial \Omega,\end{cases}
$$

where $g_{m}: \Omega \rightarrow \mathbb{R}$ form a collection of nonnegative continuous functions. If $g_{m} \rightarrow 0$, uniformly on compacta, then $\left\|v_{m}\right\| \rightarrow 0$.

Proof. Let $B$ be a ball of radius $r>0$ such that $\Omega \subset \subset B$. Define constants $C_{m}$ by $C_{m}=\sup _{\Omega} g_{m}$ and let $w_{m}=T_{k}\left(C_{m}\right)$ denote the unique solution to

$$
\begin{cases}S_{k}\left(D^{2} w_{m}\right)=C_{m} & x \in B, \\ w_{m}=0 & x \in \partial B .\end{cases}
$$

By Theorem 1.10, we must have $w_{m}(x) \leq v_{m}(x)$ for all $x \in \Omega$. Since $C_{m} \rightarrow 0$, the continuity of $T_{k}$ implies $\left\|w_{m}\right\| \rightarrow 0$, in which case $v_{m} \rightarrow 0$ for all $x \in \Omega$. The result follows.

The next lemma yields bounds on the values of $\lambda$ for solutions to (5.2). 
LEMma 5.3. Let $(\lambda, u)$ solve (5.2) with $u \neq 0$. Then $|\lambda| \leq \lambda_{0}$.

Proof. Suppose $(\lambda, u)$ is a solution of (5.2) with $|\lambda|>\left|\lambda_{0}\right|$. Let $u_{0}$ be an eigenfunction corresponding to the principal eigenvalue for the $k$-Hessian operator; i.e., $u_{0}$ satisfies the equation

$$
\begin{cases}S_{k}\left(D^{2} u_{0}\right)=\left|\lambda_{0} u_{0}\right|^{k} & x \in \Omega, \\ u_{0}=0 & x \in \partial \Omega .\end{cases}
$$

By scaling if necessary, we may assume $u(x)<u_{0}(x)$ for all $x \in \Omega$. Let $\delta^{*}>1$ be maximal such that $\left(u-\delta^{*} u_{0}\right)(x) \leq 0$ in $\Omega$. Let $L_{k}$ be the operator defined by (3.6), where $w=\delta^{*} u_{0}$. By Lemma 1.7 we have

$$
\begin{aligned}
L_{k}\left(u-\delta^{*} u_{0}\right) & \geq F_{k}\left(D^{2} u\right)-F_{k}\left(D^{2} w\right)=\left[|\lambda u|^{k}+g(u)\right]^{1 / k}-\left[\left|\lambda_{0} \delta^{*} u_{0}\right|^{k}\right]^{1 / k} \\
& =|\lambda \| u|\left[1+\frac{g(u)}{|\lambda u|^{k}}\right]^{1 / k}-\left|\lambda_{0}\right|\left|\delta^{*} u_{0}\right| \geq|\lambda||u|-\left|\lambda_{0}\right|\left|\delta^{*} u_{0}\right| \geq 0,
\end{aligned}
$$

since $|\lambda|>\left|\lambda_{0}\right|$ and $0<\left|\delta^{*} u_{0}\right| \leq|u|$ for all $x \in \Omega$. This implies, by the maximum principle, that $u=\delta^{*} u_{0}$ for all $x \in \Omega$. Therefore, $S_{k}\left(D^{2} u\right)=S_{k}\left(D^{2} w\right)$, and

$$
|\lambda u|^{k}+g(u)=\left|\lambda_{0} \delta^{*} u_{0}\right|^{k}=\left|\lambda_{0} u\right|^{k} .
$$

Hence

a contradiction, as $|\lambda|>\left|\lambda_{0}\right|$.

$$
|\lambda|^{k}-\left|\lambda_{0}\right|^{k}=-\frac{g(u)}{|u|^{k}} \leq 0,
$$

As in Section 4, we shall find the existence of continua of nontrivial solutions to (5.2) will depend on the assumptions made on $g$, either near the origin, or "near infinity." However, in the absence of the multiplicative factor of $\lambda$, we shall need to add conditions on $g$ both near the origin and near infinity.

There are two models for the nonlinear term $g$, which we shall henceforth denote by $g_{0}$ and $g_{\infty}$. We assume both $g_{0}$ and $g_{\infty}$ are nonnegative continuous functions, with the asymptotic behavior defined by

(1) $g_{0}=o\left(|u|^{k}\right)$ as $|u| \rightarrow 0$,

(2) $g_{0}(u) /|u|^{k} \rightarrow \infty$ as $|u| \rightarrow \infty$,

(3) $g_{\infty}=o\left(|u|^{k}\right)$ as $|u| \rightarrow \infty$,

(4) $g_{\infty}(u) /|u|^{k} \rightarrow \infty$ as $|u| \rightarrow 0$,

(5) $g_{\infty}$ is nondecreasing.

For example, we may take $g_{0}(u)=|\delta u|^{p}$ for any $p>k$, and $g_{\infty}(u)=|\delta u|^{p}$ for any $0 \leq p<k$, where $\delta \in \mathbb{R}$.

For either $g=g_{0}$ or $g=g_{\infty}$, we may introduce the Nemytskiu operator $N_{k}: \mathbb{R} \times E \rightarrow E^{+}$defined by

$$
N_{k}(\xi, u)=|\xi u|^{k}+g(u),
$$


and let $H_{k}=T_{k} \circ N_{k}$ denote the composition. Thus $z=H_{k}(\lambda, u)$ solves

$$
\begin{cases}S_{k}\left(D^{2} z\right)=|\lambda u|^{k}+g(u) & x \in \Omega, \\ z=0 & x \in \partial \Omega .\end{cases}
$$

Define $h_{k}: \mathbb{R} \times E \rightarrow E$ by

$$
h_{k}(\lambda, w)=w-H_{k}(\lambda, w) .
$$

If $(\lambda, u)$ is a zero of (5.4), then $u$ is a $k$-convex function which is an admissible solution to (5.2). We are now ready to state and prove some results concerning the existence of global bifurcation continua for (5.2). Let us first consider the case of $g=g_{\infty}$.

THEOREM 5.4. For $g=g_{\infty}$, the constant $\mu$ is a bifurcation value at infinity for (5.4) if and only if $|\mu|=\lambda_{0}$. Furthermore, there exists an unbounded continuum of solutions to (5.4), which lies in the strip $\left\{(\lambda, u):-\lambda_{0} \leq \lambda \leq \lambda_{0}\right\}$.

Proof. The existence of such a continuum will follow from Theorem 4.3, provided there exist constants $\lambda_{a}, \lambda_{b} \geq 0$, and $M>0$, such that all solutions to

$$
\begin{cases}S_{k}\left(D^{2} u\right)=|\lambda u|^{k}+g_{\infty}(u) & x \in \Omega, \\ u=0 & x \in \partial \Omega,\end{cases}
$$

satisfy $\|u\| \leq M$, for all $u \in E$, when $\lambda \in\left\{\lambda_{a}, \lambda_{b}\right\}$. In addition, we need the unequal degree condition

$$
\begin{aligned}
& d\left(\mathrm{id}-T_{k}\left(\left|\lambda_{a} u\right|^{k}+g_{\infty}(u)\right), B_{R}(0), 0\right) \\
& \quad \neq d\left(\mathrm{id}-T_{k}\left(\left|\lambda_{b} u\right|^{k}+g_{\infty}(u)\right), B_{R}(0), 0\right)
\end{aligned}
$$

to hold for all $R>M$.

First, we show that for any positive constant $\mu \neq \lambda_{0}$, the solutions to (5.5) are bounded. For if not, then there exists a sequence $\left\{u_{m}\right\}$ of $k$-convex solutions to (5.5) with $\lambda=\mu$, such that $\left\|u_{m}\right\| \rightarrow \infty$. By dividing through by $\left\|u_{m}\right\|^{k}$, we obtain the equation

$$
S_{k}\left(D^{2} v_{m}\right)=\left|\mu v_{m}\right|^{k}+g_{\infty}\left(u_{m}\right) /\left\|u_{m}\right\|^{k},
$$

where $v_{m}=u_{m} /\left\|u_{m}\right\|$. By properties (3) and (5) of $g_{\infty}$, the right-hand side of equation (5.7) is bounded. Therefore, by the complete continuity of $T_{k}$, the sequence $\left\{v_{m}\right\}$ contains a convergent subsequence, whose limit must correspond to the principal eigenvalue for the $k$-Hessian operator; thus $|\mu|=\lambda_{0}$.

Next, we need to verify the unequal degree condition (5.6). The same argument above shows that for balls of large enough radius, we may ignore the $g_{\infty}(u)$ term for purposes of degree calculation. Thus, one sees that condition (5.6) is equivalent to showing

$$
d\left(\mathrm{id}-\lambda_{a} A_{k}(\cdot), B_{R}(0), 0\right) \neq d\left(\mathrm{id}-\lambda_{b} A_{k}(\cdot), B_{R}(0), 0\right),
$$


for all $R>0$, sufficiently large. However, this is precisely the conclusion of Lemma 4.4.

Therefore, by Theorem 4.3, there exists a continuum $\mathcal{C}^{\infty}$ of solutions to $(5.5)$ that is unbounded in $\left[\lambda_{a}, \lambda_{b}\right] \times E$ and is either

(1) unbounded in the $\lambda$ direction, or else,

(2) there exists an interval $[c, d]$ such that $\left(\lambda_{a}, \lambda_{b}\right) \cap(c, d)=\emptyset$ and $\mathcal{C}^{\infty}$ bifurcates from infinity in $[c, d] \times E$.

By Lemma 5.3, the continuum is bounded in the $\lambda$ direction; hence, hence must also bifurcate to infinity at $-\lambda_{0}$.

In the dual setting, where $g=g_{0}$, we have the following lemma.

LEMMA 5.5. For $g=g_{0},(0,0)$ is not a bifurcation point for (5.4).

ProOF. If $(0,0)$ is a bifurcation point for $(5.4)$, then by the homogenization procedure we obtain a sequence of unit vectors $\left\{v_{m}\right\}$ satisfying

$$
S_{k}\left(D^{2} v_{m}\right)=\left|\lambda_{m} v_{m}\right|^{k}+\frac{g_{0}\left(u_{m}\right)}{\left\|u_{m}\right\|^{k}} .
$$

We may rewrite the right-hand side of (5.8) as

$$
\left|\lambda_{m} v_{m}\right|^{k}+\phi_{k}\left(u_{m}\right)\left|v_{m}\right|^{k}
$$

Consequently, by property (1) of $g_{0}$, the right-hand side of (5.8) tends to 0 as $m \rightarrow \infty$, and by Lemma 5.2 , we may conclude $\left\|v_{m}\right\| \rightarrow 0$; a contradiction, as $\left\|v_{m}\right\|=1$ for all $m$.

THEOREM 5.6. For $g=g_{0}$, the point $(\mu, 0)$ is a bifurcation point for $(5.4)$ if and only if $|\mu|=\lambda_{0}$. Furthermore, there exists a global continuum of nontrivial solutions to (5.4) which bifurcates from $\left(\lambda_{0}, 0\right)$ and lies in the strip $\{(\lambda, u)$ : $\left.-\lambda_{0} \leq \lambda \leq \lambda_{0}\right\}$

ProOF. By the same arguments employed in Theorem 4.5, together with Lemma 5.5 , it is readily established that $\left( \pm \lambda_{0}, 0\right)$ are the only possible bifurcation points for (5.4). Similarly, it is readily shown that Theorem 4.2 applies in this setting, establishing that the points $\left( \pm \lambda_{0}, 0\right)$ are bifurcation points for $(5.4)$. Thus, there exists a continuum of nontrivial solutions to (5.2), which bifurcates from $\left(\lambda_{0}, 0\right)$ and is either unbounded in $\mathbb{R} \times E$ or meets another bifurcation point, which in our case, must be $\left(-\lambda_{0}, 0\right)$. However, by Lemma 5.3 , the continuum must be bounded in $\mathbb{R}$. This completes the proof.

Therefore, we have established the existence of global branches of nontrivial solutions to (5.2) in both cases $g=g_{0}$ and $g=g_{\infty}$. We next refine the behavior of these continua in an effort to exhibit nontrivial solutions to (5.1). 
5.2. The $k$-sublinear case, $g=g_{\infty}$. In this section we refine our analysis of the global continua to (5.2) in the sublinear case $g=g_{\infty}$. We begin with a lemma.

Lemma 5.7. Let $(\lambda, u)$ correspond to a solution of (5.4) with $u \neq 0$. Then there exists $\varepsilon>0$ such that $\|u\| \geq \varepsilon$.

Proof. If not, then we may construct a sequence $\left(\lambda_{m}, u_{m}\right) \rightarrow(\mu, 0)$, such that

$$
S_{k}\left(D^{2} u_{m}\right)=\left|\lambda_{m} u_{m}\right|^{k}+g_{\infty}\left(u_{m}\right)
$$

By homogenization, we obtain unit vectors $v_{m}$ satisfying

$$
S_{k}\left(D^{2} v_{m}\right)=\left|\lambda_{m} v_{m}\right|^{k}+\phi_{k}\left(u_{m}\right)\left|v_{m}\right|^{k} \geq \phi_{k}\left(u_{m}\right)\left|v_{m}\right|^{k} .
$$

Let $\Omega^{\prime} \subset \subset \Omega$. On $\Omega^{\prime}$ the functions $v_{m}=u_{m} /\left\|u_{m}\right\|$ are bounded away from 0 and the functions $u_{m} \rightarrow 0$ uniformly; hence by property (4) of $g_{\infty}$, the functions $\phi_{k}\left(u_{m}\right) \rightarrow \infty$ uniformly on $\Omega^{\prime}$, as $m \rightarrow \infty$. Therefore, by Lemma 5.1 we must have $\left\|v_{m}\right\| \rightarrow \infty$, a contradiction, as $\left\|v_{m}\right\|=1$ for all $m \in \mathbb{N}$.

We now turn to the statement and proof of the existence theorem for (5.1) when $g=g_{\infty}$ (see Figure 5.1).

Theorem 5.8. Let $k \in\{1, \ldots, n\}$, and suppose $\Omega$ is a strictly $(k-1)$-convex domain in $\mathbb{R}^{n}$. Then there exists a global continuum of nontrivial solutions to (5.17), which crosses the $\lambda=0$ axis nontrivially. Therefore, the equation

$$
\begin{cases}S_{k}\left(D^{2} u\right)=g_{\infty}(u) & x \in \Omega \\ u=0 & x \in \partial \Omega\end{cases}
$$

has a nontrivial $k$-convex solution.

Proof. The existence of the global continuum of nontrivial solutions follows from Theorem 5.4. By Lemma 5.7, the continuum cannot pass through the origin. Furthermore, by Theorem 5.4, it also follows that 0 is not an asymptotic bifurcation value for (5.17). Therefore, the continuum must cross the $\lambda=0$ axis nontrivially, demonstrating that (5.9) has a nontrivial $k$-convex solution.

As an application, we obtain a nontrivial solution to the $k$-Hessian equation

$$
\begin{cases}S_{k}\left(D^{2} u\right)=|\delta u|^{p} & x \in \Omega, \\ u=0 & x \in \partial \Omega,\end{cases}
$$

for all $\delta \in \mathbb{R}$ and $0 \leq p<k$. Moreover, we can also conclude uniqueness:

THEOREM 5.9. There exists a unique nontrivial solution to (5.10).

Proof. The essential ingredients in the proof are due to Oliker [33], who considered the case $k=n$ and $g(u)=|u /(n-1)|$ in $\mathbb{R}^{n}(n \geq 2)$. Suppose $u, v$ 


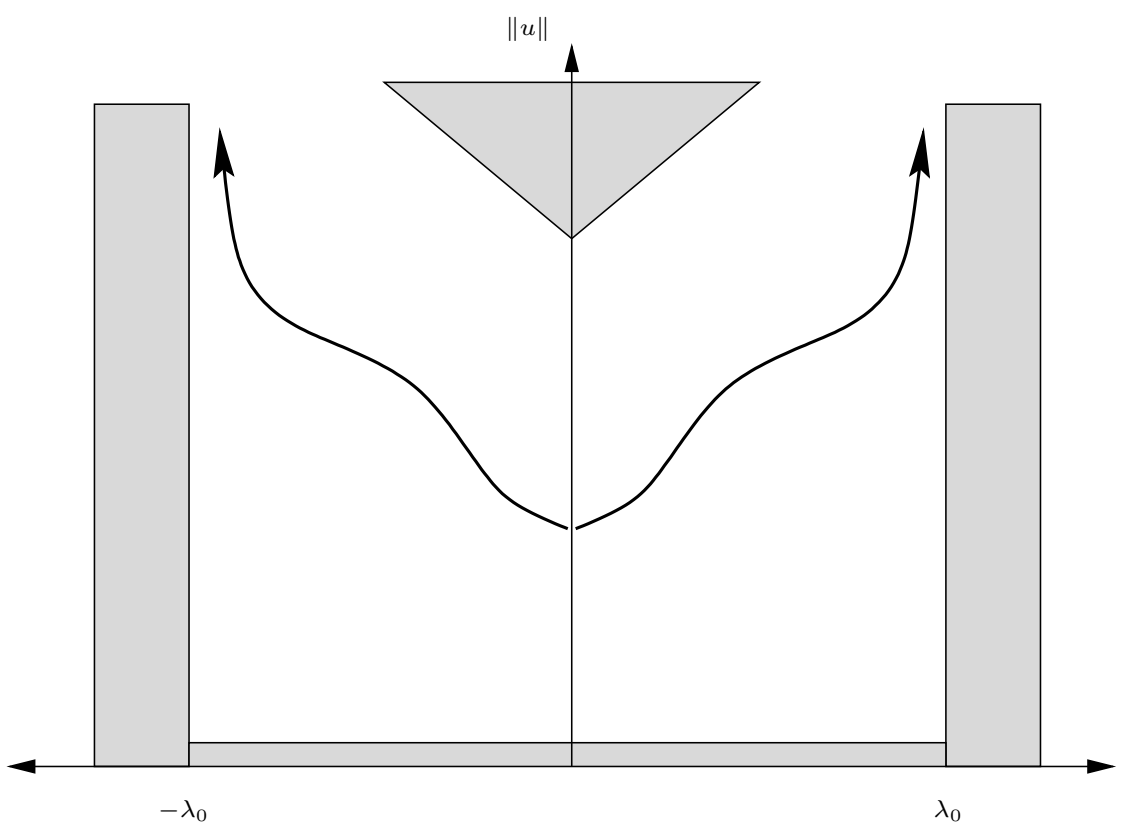

Figure 5.1. Possible continuum for (5.2) in the $k$-sublinear case

are two nontrivial admissible solutions to (5.10), with $u \neq v$. By relabeling if necessary, we may assume that there exist points in $\Omega$ such that $v(x)<u(x)$. Let $\delta_{*}>0$ be maximal such that $u(x) \leq\left(\delta_{*} v\right)(x)<0$ for all $x \in \Omega$. Note that $\delta_{*}<1$ and there exists $x_{0} \in \Omega$ such that $u\left(x_{0}\right)=\delta_{*} v\left(x_{0}\right)$. The computation

$$
S_{k} D^{2}\left(\delta_{*}^{p / k} v\right)=\delta_{*}^{p} S_{k}\left(D^{2} v\right)=\delta_{*}^{p}|\delta v|^{p} \leq|\delta u|^{p}=S_{k}\left(D^{2} u\right),
$$

implies that $u(x) \leq\left(\delta_{*}^{p / k} v\right)(x)$ for all $x \in \Omega$. In particular,

$$
\delta_{*} v\left(x_{0}\right)=u\left(x_{0}\right) \leq\left(\delta_{*}^{p / k} v\right)\left(x_{0}\right) .
$$

However, if $p<k, \delta_{*}<1$, and $v\left(x_{0}\right)<0$, then $\delta_{*}^{p / k}>\delta_{*}$ and

$$
\delta_{*} v\left(x_{0}\right)=u\left(x_{0}\right) \leq \delta_{*}^{p / k} v\left(x_{0}\right)<\delta_{*} v\left(x_{0}\right),
$$

a contradiction. Thus, $u=v$.

Theorems of this type for the $k$-Hessian operators were first considered by Tso [47], where solutions are established in the radially symmetric case ( $\Omega$ a ball) using variational methods. This theorem extends the existence result of [47] (in the case $p<k$ ) to the class of $k$-convex domains, however, it is worth remarking that the existence results of [47] also include the cases where $k<p<\gamma(k)$, which are not available at this time, via these methods. 
As mentioned in the introduction, this theorem also includes a results due to Oliker [33], in the case $k=n, g(u)=|u /(n-1)|$, and $\Omega$ is a bounded, strictly convex domain in $\mathbb{R}^{n}$, with $n \geq 2$.

5.3. The $k$-superlinear case, $g=g_{0}$. The behavior of the continua to (5.2) in the $k$-superlinear case turns out to be a bit more delicate. This distinction becomes apparent when one considers the case $k=1$ and $g(u)=u^{p}$ for $p$ large. The well-known nonexistence results of Pohozaev [37] demonstrate that the continua of solutions to (5.2) may not cross the $\lambda=0$ axis nontrivially.

In the $k$-sublinear case, we make assumptions on $g_{\infty}$ near infinity to obtain a global branch of solutions to (5.2) which bifurcates to infinity at $\lambda_{0}$. We then use information about $g_{\infty}$ near the origin to get the branch to turn. In the $k$ superlinear case, we make assumptions on $g_{0}$ near the origin to obtain bifurcation from the trivial branch and then use information about $g_{0}$ near infinity to get the branch to bend. These estimates near infinity provide the delicacy in these arguments. This is best illustrated by first considering the Monge-Ampère case.

5.3.1. The Monge-Ampère case. Let us now consider the Monge-Ampère case of (5.1) defined by

$$
\begin{cases}\operatorname{det} D^{2} u=g_{0}(u) & x \in \Omega, \\ u=0 & x \in \partial \Omega,\end{cases}
$$

We begin with some auxiliary lemmas. The first lemma is a basic estimate for convex functions.

Lemma 5.10. $\Omega$ be a convex domain in $\mathbb{R}^{n}$. If $u: \bar{\Omega} \rightarrow \mathbb{R}$ is a convex function such that $\left.u\right|_{\partial \Omega}=0$, then the inequality

$$
|u(x)| \geq \frac{\operatorname{dist}(x, \partial \Omega)}{\operatorname{diam}(\Omega)}\|u\|
$$

holds for all $x$ in $\Omega$, where $\|u\|$ is the supremum norm of $u \in C(\bar{\Omega})$.

Proof. The inequality is trivial in the case $\|u\|=0$; thus let us assume $\|u\| \neq 0$. It follows from the convexity of $\Omega$ that $u \in C(\Omega)$; thus since $\left.u\right|_{\partial \Omega}=0$, there must exist $z \in \Omega$ such that $u(z)=\min _{\Omega} u=-\|u\|<0$. Let $\Gamma$ denote the geometric cone in $\mathbb{R}^{n+1}$ with vertex $(z, u(z))$ and base $\partial \Omega$. Let $\gamma: \bar{\Omega} \rightarrow \mathbb{R}$ represent the function whose graph is $\Gamma$. By convexity of $u$, we have $u(x) \leq \gamma(x)$ for all $x \in \bar{\Omega}$.

Let $x \in \Omega \backslash\{z\}$ be arbitrary and consider the line segment starting at $z$, passing through $x$, hitting $\partial \Omega$ at the point $\eta=\eta(x)$. By considering similar triangles in the plane defined by this line segment and the point $(z, u(z))$, we obtain

$$
\frac{\gamma(x)}{u(z)}=\frac{\operatorname{dist}(x, \eta)}{\operatorname{dist}(z, \eta)}
$$


Since $\operatorname{dist}(x, \partial \Omega) \leq \operatorname{dist}(x, \eta)$ and $\operatorname{dist}(z, \eta) \leq \operatorname{diam}(\Omega)$, we have

$$
\frac{\operatorname{dist}(x, \partial \Omega)}{\operatorname{diam}(\Omega)} \leq \frac{\operatorname{dist}(x, \eta)}{\operatorname{diam}(\Omega)} \leq \frac{\operatorname{dist}(x, \eta)}{\operatorname{dist}(z, \eta)}=\frac{\gamma(x)}{u(z)} .
$$

Multiplying by $u(z)$ and recalling $u(z)<0$ we obtain

$$
u(z) \frac{\operatorname{dist}(x, \partial \Omega)}{\operatorname{diam}(\Omega)} \geq \gamma(x) .
$$

Finally, as $-u(z)=\|u\|$, we have

$$
|u(x)|=-u(x) \geq-\gamma(x) \geq \frac{\operatorname{dist}(x, \partial \Omega)}{\operatorname{diam}(\Omega)}\|u\|,
$$

which is the desired result.

As a consequence of this we have the following lemma:

Lemma 5.11. Let $\Omega$ be a bounded, convex domain in $\mathbb{R}^{n}$. Let $\left\{u_{m}\right\} \subset C(\bar{\Omega})$ be a sequence of convex functions with $\left.u_{m}\right|_{\partial \Omega}=0$ for all $k=1,2, \ldots$ Furthermore, suppose $\left\|u_{m}\right\| \rightarrow \infty$. Then $\left|u_{m}\right| \rightarrow \infty$ uniformly on compacta; that is, uniformly on compact subsets of $\Omega$.

The a priori convexity of solutions to (5.2) in the Monge-Ampère case provide the key to the following lemma, which is essential to our analysis of (5.11).

LEMma 5.12. If $(\lambda, u)$ is a zero of (5.4), then there exists $M>0$, sufficiently large such that $\|u\| \leq M$.

Proof. If not, then there exists a sequence $\left\{\left(\lambda_{m}, u_{m}\right)\right\}$, with $\left\|u_{m}\right\| \rightarrow \infty$. By Lemma 5.3, the sequence $\left\{\lambda_{m}\right\} \subset\left[-\lambda_{0}, \lambda_{0}\right]$; hence, without loss of generality, we may assume $\lambda_{m} \rightarrow \mu \in\left[-\lambda_{0}, \lambda_{0}\right]$. In other words, $\mu$ is an asymptotic bifurcation value for (5.4). By homogenization, we obtain unit vectors $v_{m}=u_{m} /\left\|u_{m}\right\|$ satisfying the equation

$$
\operatorname{det} D^{2} v_{m}=\left|\lambda_{m} v_{m}\right|^{n}+\frac{g_{0}\left(u_{m}\right)}{\left\|u_{m}\right\|^{n}} \geq \frac{g_{0}\left(u_{m}\right)}{\left\|u_{m}\right\|^{n}} .
$$

As before, we may rewrite the right-hand side of (4.11) as

$$
\frac{g_{0}\left(u_{m}\right)}{\left\|u_{m}\right\|^{n}}=\phi_{n}\left(u_{m}\right)\left|v_{m}\right|^{n}
$$

where $\phi_{n}$ is defined by (5.13). Let $\Omega^{\prime} \subset \subset \Omega$. On $\Omega^{\prime}$ the functions $v_{m}$ are bounded away from 0 and by Lemma 5.11 , the functions $u_{m}$ satisfy $\left|u_{m}\right| \rightarrow \infty$ uniformly. Therefore, by property (2) of $g_{0}$, the functions $\phi_{n}\left(u_{m}\right) \rightarrow \infty$ uniformly on $\Omega^{\prime}$ as $m \rightarrow \infty$. Combining (5.13) with Lemma 5.1 we may conclude $\left\|v_{m}\right\| \rightarrow \infty$, which yields a contradiction as $\left\|v_{m}\right\|=1$.

It is instructive to see where this proof fails in the analogous situation considered previously in Theorem 4.5 in Section 4. Namely, in that section, there 
was a multiplicative factor of $\lambda_{m}$ in front of the term $\phi_{n}\left(u_{m}\right)$, in which case the above argument would fail if $\lambda_{m} \rightarrow 0$ as $\left\|u_{m}\right\| \rightarrow \infty$, which is precisely the case.

The ideas above establish the existence of a nontrivial solution to (5.11) (see Figure 5.2):

THEOREM 5.13. The global bifurcation branch crosses the $\lambda=0$ axis nontrivially.

Proof. By Theorem 5.6, there exists a continuum of nontrivial solutions to (5.2) which bifurcates from $\left(\lambda_{0}, 0\right)$. The a priori bounds of Lemma 5.12 establish that the continuum must cross the axis $\lambda=0$ nontrivially.

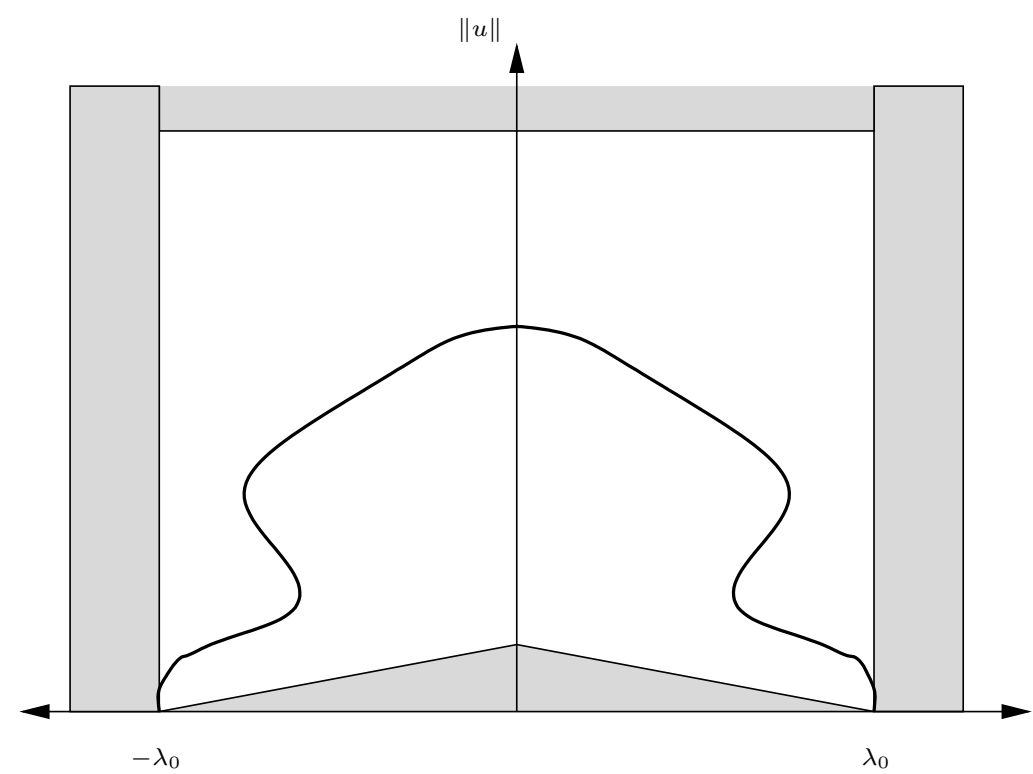

Figure 5.2. Possible continuum for (5.2) in the $n$-superlinear case

As one application of this theorem, we obtain a nontrivial solution to the equation

$$
\begin{cases}\operatorname{det} D^{2} u=|\delta u|^{p} & x \in \Omega, \\ u=0 & x \in \partial \Omega,\end{cases}
$$

for all $\delta \in \mathbb{R}$ and $p>n$. In particular, we see that there is no critical exponent for the Monge-Ampère operator. Solutions to problems of this type have been previously established using variational methods by Tso [46].

By combining the results of the previous sections, we obtain the following concise theorem for Monge-Ampère equations: 
ThEOREM 5.14. Let $\Omega \subset \mathbb{R}^{n}$ be a strictly convex, bounded domain. Then the Monge-Ampère equation

$$
\begin{cases}\operatorname{det} D^{2} u=|\delta u|^{p} & x \in \Omega, \\ u=0 & x \in \partial \Omega,\end{cases}
$$

has a nontrivial admissible solution for all $p \neq n, p \geq 0$, and $\delta \in \mathbb{R}$. In the case $p<n$, the solution is unique. Furthermore, in the case $p=n,(5.15)$ has a line of solutions corresponding to the unique eigenvalue $\delta=\lambda_{0}>0$.

5.3.2. The general case $1 \leq k<n$. Let us now consider the general case of (5.1) defined by

$$
\begin{cases}S_{k}\left(D^{2} u\right)=g_{0}(u) & x \in \Omega, \\ u=0 & x \in \partial \Omega .\end{cases}
$$

By Theorem 5.6, there exists a global branch of nontrivial solutions to the equation

$$
\begin{cases}S_{k}\left(D^{2} u\right)=|\lambda u|^{k}+g_{0}(u) & x \in \Omega, \\ u=0 & x \in \partial \Omega .\end{cases}
$$

However, we no longer have precise control on the long-term behavior of the continua. For instance, we may no longer conclude a continuum is bounded in $E$ (see Lemma 5.12). The essential ingredient that allowed us to bound the continuum in $E$ was the convexity of the solutions $u_{k}$, which in particular, allowed us to appeal to Lemma 5.11, which would force the nonlinear perturbations to vanish in the limit. Thus in the general case $1 \leq k<n$, the continuum may bifurcate to infinity, in which case we may not conclude the bifurcation branch crosses the $\lambda=0$ axis nontrivially. In fact, for certain cases (e.g. $k=1$, $g(u)=u^{p}$ for $\left.p \geq(n+2) /(n-2)\right)$, the continuum must not cross the $\lambda=0$ axis nontrivially, in which case we may conclude (5.16) has an asymptotic bifurcation value $0 \leq \mu \leq \lambda_{0}$. This contrast further implies that in $\mathbb{R}^{n}$ for $n \geq 3$, there exists a sequence of 1-convex (continuous and subharmonic) functions such that $\left\|u_{k}\right\| \rightarrow \infty$, but $\left|u_{k}\right|$ does not converge to infinity, uniformly on compacta.

For any values of $k$ such that the $k$-convex functions satisfy Lemma 5.11, then the techniques above would allow one to establish the same conclusions as Theorem 5.13. In particular, the continuum will cross the $\lambda=0$ axis nontrivially. Hence we are led to the natural question: For which values of $k$ do the $k$-convex functions satisfy the convergence property of Lemma 5.11? Furthermore, one has the directly related question: For which values of $k$ does the $k$-Hessian operator have a critical exponent?

In [47], Tso uses an identity due to Pucci and Serrin [38] to answer the second question: 
Theorem 5.15 (Tso [47]). Let $\Omega$ be a ball and let $\gamma(k)$ be defined by

$$
\gamma(k)= \begin{cases}\frac{(n+2) k}{n-2 k} & 1 \leq k<n / 2 \\ \infty & n / 2 \leq k \leq n\end{cases}
$$

Then (5.16) has no admissible solution $u \in C^{1}(\bar{\Omega}) \cap C^{4}(\Omega)$ when $p \geq \gamma(k)$.

Recently, the authors of [9] use similar techniques, attributed to Rellich [39] and Pohozaev [37], to prove a similar nonexistence result for a large class of ordinary differential operators, which includes the radial case of the $k$-Hessian and the $p$-Laplace operators.

Corresponding existence results are also shown in [47], [8], in the complementary case $p<\gamma(k)$, in harmony with our results in the previous section.

One interesting outcome of this, which is obtained by mixing the nonexistence result above with our techniques, is the following proposition which sheds some light onto the geometry of $k$-convex functions:

Proposition 5.16. Let $1 \leq k<n / 2$. Then there exists a sequence of (continuous) $k$-convex functions such that $\left\|u_{k}\right\| \rightarrow \infty$, but the functions $\left|u_{k}\right| d o$ not tend to infinity uniformly on compacta.

It is not clear at this time if this proposition fails in the remaining cases $n / 2 \leq k<n$. However, the nonexistence of critical exponents in these cases provides strong evidence on its behalf. It is also not clear at this time how to determine the critical exponents via our technique. The evidence above points to developing a better understanding of $k$-convex functions in the intermediate cases. For instance, there is strong evidence for the following conjecture:

Conjecture 5.17. For each integer $k$ with $n / 2 \leq k \leq n$, if $\left\{u_{m}\right\}$ is a sequence of $k$-convex functions in $E$ such that $\left\|u_{m}\right\| \rightarrow \infty$, then $\left|u_{m}\right| \rightarrow \infty$ uniformly on compacta.

If this conjecture is true, then our techniques will establish the existence of a nontrivial solution to (5.16) for any $k \geq n / 2$ and $p>0$ with $p \neq n$. However, at this time, it remains to prove this conjecture.

\section{Liouville-Gelfand problem}

The classical Liouville-Gelfand problem is the following boundary value problem:

$$
\begin{cases}-\Delta u=\lambda e^{u} & x \in \Omega, \\ u>0 & x \in \Omega, \\ u=0 & x \in \partial \Omega\end{cases}
$$


for $\lambda \in \mathbb{R}$. If $\Omega=B_{R}(0)$ is the open ball of radius $R$ centered at the origin in $\mathbb{R}^{n}$, then by the well-known results of Gidas-Ni-Nirenberg [14], all possible solutions to (6.1) are radially symmetric; hence (6.1) becomes the ordinary differential equation

$$
\begin{cases}-u^{\prime \prime}-\frac{n-1}{r} u^{\prime}=\lambda e^{u} & r \in(0, R), \\ u>0 & r \in[0, r), \\ u^{\prime}(0)=u(R)=0 . & \end{cases}
$$

This problem was studied by Liouville [30] in the case $n=1$, Bratu [4] in the case $n=2$, and later, Gelfand [13] for higher dimensions.

Of particular interest is the relation between dimension and multiplicity results for (6.2) observed by Joseph and Lundgren [20] which we recalled in the introduction.

6.1. The Monge-Ampère case. Here we consider the analogous problem to (6.1) for the Monge-Ampère operator; namely, we consider the equation

$$
\begin{cases}\operatorname{det} D^{2} u=\lambda e^{-u} & x \in \Omega, \\ u=0 & x \in \partial \Omega,\end{cases}
$$

on a strictly convex bounded domain $\Omega \subset \mathbb{R}^{n}$. The choice of $e^{-u}$ is natural as solutions to the general Monge-Ampère equation with Dirichlet boundary conditions are negative in the elliptic case. However, one can also consider the choice of $e^{u}$ and obtain similar results.

We will show that, in contrast to the problem (6.1), the qualitative nature of the solution continua for (6.3) does not depend on the space dimension $n$. This will use, in an essential way, the convexity of solutions to elliptic Monge-Ampère equations.

Define $G: \mathbb{R} \times E \rightarrow E$ by $G(\lambda, u)=|\lambda|^{1 / n} T_{n}\left(e^{-u}\right)$, where $T_{n}\left(e^{-u}\right)=z$ is the unique admissible solution to the equation

$$
\begin{cases}\operatorname{det} D^{2} z=e^{-u} & x \in \Omega, \\ z=0 & x \in \partial \Omega .\end{cases}
$$

The compactness of the solution operator $T_{n}$ for elliptic Monge-Ampère equations (Proposition 3.2) implies the mapping $G$ is completely continuous on $\mathbb{R} \times E$. Furthermore as $G(0, \cdot)=0$, we have

$$
d\left(\mathrm{id}-G(0, \cdot), B_{r}(0), 0\right)=d\left(\mathrm{id}, B_{r}(0), 0\right)=1,
$$

for any $r>0$. Therefore by the continuum property of the Leray-Schauder degree (see Theorem 4.1), there exists an unbounded continuum of solutions to

$$
u-G(\lambda, u)=0,
$$


whose components satisfy (6.3). Note that $u=0$ is the unique solution corresponding to $\lambda=0$; hence the continuum cannot meet the axis $\lambda=0$ except at the point $(0,0)$. Notice also, that for $\lambda>0$, the solution $u=0$ is not a solution to $(6.3)$.

We seek to further refine the behavior of this continuum. First, we show the continuum is bounded in the $\lambda$ direction.

Lemma 6.1. Let $(\lambda, u)$ be a solution of (6.3) with $\lambda>0$. Then $\lambda \leq n ! \lambda_{0}^{n}$, where $\lambda_{0}$ is the principal eigenvalue associated with the Monge-Ampère operator for the domain $\Omega$.

Proof. Assume $(\lambda, u)$ is a solution of $(6.5)$ and let $u_{0}$ be an eigenfunction for $\lambda_{0}$. By scaling if necessary, we may assume $u(x)<u_{0}(x)$ for all $x \in \Omega$. Let $\delta^{*}$ be maximal such that $u-\delta^{*} u_{0} \leq 0$ for all $x \in \Omega$ and consider the linear second order elliptic operator

$$
L=F_{i j}\left(D^{2} w\right) D_{i j}
$$

where $w=\delta^{*} u_{0}$. As $w \in \Phi^{n}(\Omega)$, we may apply Lemma 1.7 to conclude

$$
L(u-w) \geq F\left(D^{2} u\right)-F\left(D^{2} w\right)=\left[\lambda e^{-u}\right]^{1 / n}-\left[\left|\lambda_{0} w\right|^{n}\right]^{1 / n} .
$$

The inequalities

$$
e^{-u(x)} \geq e^{-w(x)} \geq(-w(x))^{n} / n !
$$

follow from the fact that $u, w$ are negative functions and $u(x) \leq w(x)$ on $\Omega$. Therefore

$$
\frac{(-w)^{n}}{n !} \leq e^{-u} \quad \text { and } \quad \lambda_{0}^{n}(-w)^{n} \leq n ! \lambda_{0}^{n} e^{-u} \leq \lambda e^{-u}
$$

which combined with (6.6), imply $L(u-w) \geq 0$. Therefore, by the maximum principle, we must have $u=w$ for all $x \in \Omega$. Since $u=w$ we have

$$
\lambda e^{-u}=\left|\lambda_{0} w\right|^{n}=\left|\lambda_{0} u\right|^{n}
$$

in which case

$$
\lambda \frac{(-u)^{n}}{n !} \leq\left|\lambda_{0} u\right|^{n}=\lambda_{0}^{n}(-u)^{n}, \quad \text { or } \quad \lambda \leq \lambda_{0}^{n} n !
$$

which completes the proof.

Next we show that in fact 0 corresponds to the unique asymptotic bifurcation value for (6.5), in which case (6.3) has at least two nontrivial solutions for all $\lambda>$ 0 , sufficiently small. It will be clear from the proof that there is no dependence on the space dimension $n$. 
Lemma 6.2. Let $I=\left[\mu, n ! \lambda_{0}^{n}\right] \subset \mathbb{R}$ for some $\mu>0$ and suppose that there exists a sequence $\left(\lambda_{m}, u_{m}\right)$ of solutions to (6.5) with $\lambda_{m} \in I$ for all $m$. Then there exists a constant $M>0$ such that $\left\|u_{m}\right\| \leq M$.

Proof. Suppose $\left(\lambda_{m}, u_{m}\right)$ is a sequence of solutions such that $\left\|u_{m}\right\| \rightarrow \infty$ and $\left\{\lambda_{m}\right\} \subset I$. As $I$ is compact, we may assume, by relabeling if necessary, that $\lambda_{m} \rightarrow \lambda \in I$. By homogenization we obtain the sequence of unit vectors $v_{m}=u_{m} /\left\|u_{m}\right\|$ such that

$$
\operatorname{det} D^{2} v_{m}=\lambda_{m} \frac{e^{-u_{m}}}{\left\|u_{m}\right\|^{n}}=\lambda_{m} \frac{e^{\left|u_{m}\right|}}{\left|u_{m}\right|^{n}}\left|v_{m}\right|^{n} .
$$

Let $\Omega^{\prime} \subset \subset \Omega$. By Lemma 5.11 (convexity of $u_{m}$ ), we have $\left|u_{m}\right| \rightarrow \infty$ on $\Omega^{\prime}$. Furthermore, on $\Omega^{\prime}$, the values of $\left|v_{m}\right|$ are bounded away from 0 . Since the values of $\lambda_{m}$ are also bounded away from 0 , the right-hand side of (6.7) must tend to $\infty$, uniformly on $\Omega^{\prime}$. Therefore, by Lemma $5.1,\left\|v_{m}\right\| \rightarrow \infty$, a contradiction as $\left\|v_{m}\right\|=1$ for all $m$.

In fact, by studying the limiting behavior of (6.7) for sequences $\left\{u_{m}\right\}$ with $\left\|u_{m}\right\| \rightarrow \infty$, one sees that we would need $\lambda_{m} \rightarrow 0$ to avoid the contradiction above, namely, $\left\|v_{m}\right\|=1$. Moreover, $\lambda$ should tend to zero like $x^{n} / e^{x}$. This remark yields the following theorem:

TheOREM 6.3. There exists $\lambda^{*}>0$ such that (6.3) has at least two solutions for all $\lambda \in\left(0, \lambda^{*}\right)$.

A possible diagram of the continuum is shown in Figure 6.1. Note that, although the quantitative results indicated by the figure hold in the case $n=1$, Theorem 6.3 does not imply that the continuum has this exact shape for $n \geq 2$, and this remains to be studied. For instance, it is natural to ask the following questions: Is there a unique solution at $\lambda=\lambda^{*}$ ? Are there precisely two solutions for each $\lambda<\lambda^{*}$ ? The precise structure of the solution set to (6.3) remains an open question, which we hope to address in the future.

By examining the proof above, it is evident that we may replace the exponential term $e^{|u|}$ by any positive continuous function $f$ satisfying

(1) $f(0)>0$,

(2) $f(u)$ is nonincreasing for $u<0$,

(3) $f(u) \geq C|u|^{n}$ for some constant $C>0$,

(4) $f(u) /|u|^{n} \rightarrow \infty$ as $|u| \rightarrow \infty$.

6.2. The general case $1<k<n$. We end with a discussion of the generalization of the Liouville-Gelfand equation (6.1) to $k$-Hessian operators

$$
\begin{cases}S_{k}\left(D^{2} u\right)=\lambda e^{-u} & x \in \Omega, \\ u=0 & x \in \partial \Omega,\end{cases}
$$




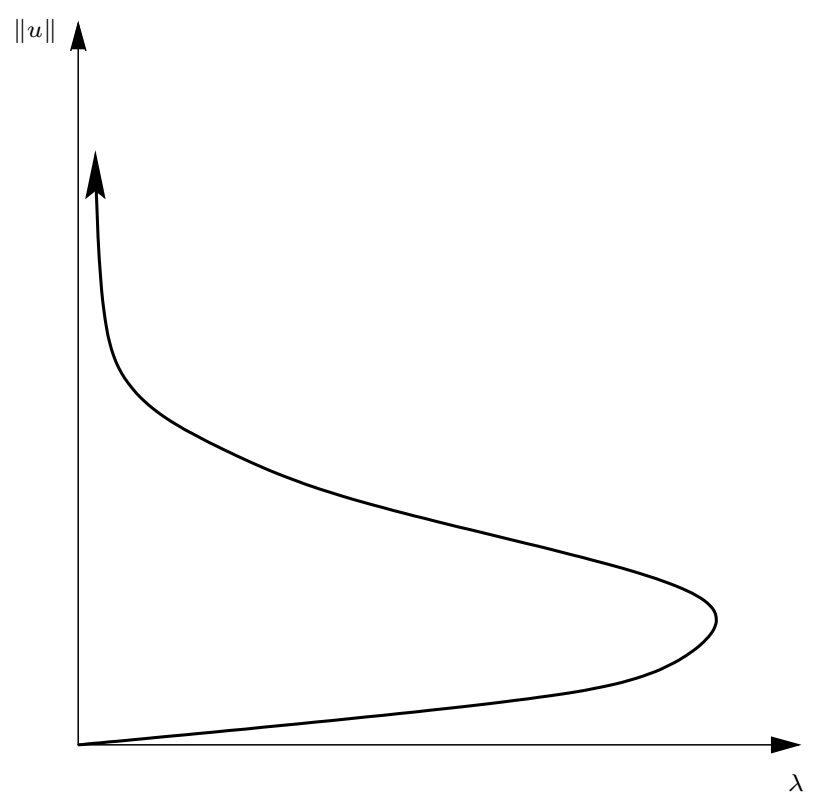

Figure 6.1. Possible continuum of solutions to (6.3)

for $\Omega \subset \mathbb{R}^{n}$, a $(k-1)$-convex domain. As above, one can readily establish that (6.8) has a global branch of nontrivial solutions $\{(\lambda, u)\}$ such that $0 \leq \lambda \leq k ! \lambda_{0}^{k}$, where $\lambda_{0}$ is the principal eigenvalue associated with the $k$-Hessian operator. The behavior for the solution continua to (6.8) is then understood in the extremal cases $k=1$ and $k=n$. However, arguments used in the case $k=n$ that used convexity will not extend to the general setting.

In [9], the authors prove that the bifurcation diagram for (6.8) in the case of radial solutions and $k=n / 2$ satisfies the same properties as (6.1) in the case $n=1$ and 2. Namely, there exists a $\lambda^{*}>0$ such that there is a unique solution at $\lambda=\lambda^{*}$ and precisely two nontrivial solutions for $\lambda \in\left(0, \lambda^{*}\right)$.

If Conjecture 5.17 is true then the methods of Section 6.1 will extend for (6.8) to establish a theorem similar to Theorem 6.3 .

The results of [9] and our results here provide strong motivation for the following conjecture:

Conjecture 6.4. For $k>n / 2$ there exists a constant $\lambda^{*}>0$, such that (6.8) has exactly one solution at $\lambda^{*}$ and precisely two solutions for $\lambda \in\left(0, \lambda^{*}\right)$.

Perhaps a more interesting desired result would be to know the precise structure of the solution continua as the parameter $k$ tends to 1 , where we know the precise dependence of the qualitative behavior of the solution continua on the space dimension $n$. 


\section{REFERENCES}

[1] A.-M. Ampère, Mémoire contenant l'application de la théorie exposée dans le XVII cahier, J. l'Ecole Polytechnique 18 (1820).

[2] I. Bakelman, Convex Analysis and Nonlinear Geometric Elliptic Equations, SpringerVerlag, Berlin, 1994.

[3] G. Boole, Ueber die partielle Differentialgleichungen 2. Ordnung, $R r+S s+T t+U(r t-$ $\left.s^{2}\right)=V$, J. Reine Angew. Math. 61 (1863), 309-333.

[4] G. Bratu, Sur les équations intégrales non linéaires, Bull. Soc. Math. France 42 (1914), 113-142.

[5] L. Caffarelli, L. Nirenberg and J. Spruck, The Dirichlet problem for nonlinear second-order elliptic equations I. Monge-Ampère equation, Comm. Pure Appl. Math. 37 (1984), 369-402.

[6] The Dirichlet problem for nonlinear second order elliptic equations III: Functions of the eigenvalues of the Hessian, Acta Math. 155 (1985), 261-301.

[7] E. CALABI, Improper affine hyperspheres of convex type and a generalization of a theorem of $K$ Jörgens, Michigan Math. J. 5 (1958), 105-126.

[8] K.-S. Chou And X. J. Wang, Variational solutions to Hessian equations, Australian National University, Mathematics Research Report (ANU MRR) 045-96 (1996).

[9] P. Clément, D. de Figueiredo and E. Mitidieri, Quasilinear elliptic equations with critical exponents, Topol. Methods Nonlinear Anal. 7 (1996), 133-170.

[10] A. Colesanti And P. Salani, Generalized solutions of Hessian equations, Bull. Austral. Math. Soc. 56 (1997), 459-466.

[11] A. DeMorgan, Cambridge Philosophical Transactions, 9. Pt. 4.

[12] L. GÅRding, An inequality for hyperbolic polynomials, J. Math. Mech. 8 (1959), 957965.

[13] I. M. Gelfand, Some problems in the theory of quasi-linear equations, Amer. Math. Soc. Transl. Ser. (2) 29 (1963), 295-381.

[14] B. Gidas, W. Ni AND L. NiREnBerg, Symmetry and related properties via the maximum principle, Comm. Math. Phys. 68 (1979), 209-243.

[15] D. Gilbarg and N. S. Trudinger, Elliptic Partial Differential Equations of Second Order, Springer-Verlag, Berlin, 1998.

[16] É. Goursat, Leçons sur l'integration des Équations aux Dérivées Partielles du Second Ordre, Hermann, Paris, 1896.

[17] L. Hörmander, Notions of Convexity, Birkhäuser Boston, Inc., Boston, MA, 1994.

[18] R. Horn and C. Johnson, Matrix Analysis, Cambridge University Press, Cambridge, 1990.

[19] J. Jacobsen, On Bifurcation Problems Associated with Monge-Ampère Operators, University of Utah, 1999.

[20] D. Joseph And T. Lundgren, Quasilinear Dirichlet problems driven by positive sources, Arch. Rational Mech. Anal. 49 (1973), 241-269.

[21] M. A. KrasnoseL'SKII, Topological Methods in the Theory of Nonlinear Integral Equations, vol. 45, Pergamon, New York, 1964.

[22] M. Krein And M. Rutman, Linear operators leaving invariant a cone in a Banach space, Amer. Math. Soc. Transl. Ser. (2) 10 (1950), 1-128.

[23] N. V. Krylov, Degenerate nonlinear elliptic equations, Mat. Sb. 120 (1983), 311-330; English transl. in Math. USSR-Sb. 48 (1984), 307-326.

[24] V. Le And K. Schmitt, Global Bifurcation in Variational Inequalities, vol. 123 in Applied Mathematical Sciences, Springer-Verlag, New York, 1997. 
[25] H. Lewy, On differential geometry in the large, I (Minkowski's Problem), Trans. Amer. Math. Soc. 43 (1938), 258-270.

[26] S. LiE, Ueber Complexe insbesondere Linien und Kugelcomplexe, mit Anwendurg auf die Theorie partieller Differential-Gleichungen, Math. Ann. 5 (1872), 145-256.

[27] Neue Integrations-methode der Monge-Ampereschen Gleichung, Ark. Mat. Nat. 1 (1876).

[28] P. L. Lions, Sur les équations de Monge-Ampère. I., Manuscripta Math. 41 (1983), $1-43$.

[29] - Two remarks on Monge-Ampère equations, Ann. Mat. Pura Appl. 142 (1985), 263-275.

[30] J. Liouville, Sur l'équation aux dérivées partielles $\frac{d^{2} \log \lambda}{d u d v} \pm 2 \lambda a^{2}=0$, J. Math. Pures Appl. 18 (1853), 71-72.

[31] H. Minkowski, Volumen and oberfläche, Math. Ann. 57 (1903), 447-495.

[32] G. Monge, Mémoire sur le calcul intégral des équations aux différences partielles, Histoire de l'Académie des Sciences, 1784.

[33] V. Oliker, Evolution of nonparametric surfaces with speed depending on curvature, $I$. The Gauss curvature case, Indiana Univ. Math. J. 40 (1991), 237-257.

[34] H. O. Peitgen And K. Schmitt, Positive and spurious solutions of nonlinear eigenvalue problems, Lecture Notes in Math., vol. 878, Springer-Verlag, 1981, pp. 275-324.

[35] Global analysis of two-parameter elliptic eigenvalue problems, Trans. Amer. Math. Soc. 283 (1984), 57-95.

[36] A. V. Pogorelov, Monge-Ampère Equations of Elliptic Type, Gröningen, Noordhoff, 1964.

[37] S. L. Pohozaev, On the eigenfunctions of the equation $\Delta u+\lambda f(u)=0$, Dokl. Akad. Nauk SSSR 6 (1965), 1408-1411.

[38] P. Pucci And J. Serrin, A general variational inequality, Indiana Univ. Math. J. 35 (1986), 681-703.

[39] F. Rellich, Darstellung der Eigenwerte von $\Delta u+\lambda u=0$ durch ein Randintegral, Math. Z. 46 (1940), 635-636.

[40] K. Schmitt, Analysis Methods for the Study of Nonlinear Equations, Lecture Notes, University of Utah, 1995.

[41] _ Positive solutions of semilinear elliptic boundary value problems, Topological Methods in Differential Equations and Inclusions (A. Granas and M. Frigon, eds.), vol. 472 of Series C: Mathematical and Physical Sciences, Kluwer Academic, 1995, pp. $447-500$.

[42] N. S. Trudinger, On the Dirichlet problem for Hessian equations, Acta Math. 175 (1995), 151-164.

[43] Weak solutions of Hessian equations, Comm. Partial Differential Equations 22 (1997), 1251-1261.

[44] N. S. Trudinger And X. J. WANG, Hessian measures I, Topol. Methods Nonlinear Anal. 10 (1997), 225-239.

[45] Hessian measures II, Australian National University, Mathematics Research Report (ANU MRR) 035-97, 1997.

[46] K. Tso, On a real Monge-Ampère functional, Invent. Math. 101 (1990), 425-448.

[47] Remarks on critical exponents for Hessian operators, Ann. Inst. H. Poincaré Anal. Non Linéaire 7 (1990), 113-122.

[48] J. URBAS, On the existence of nonclassical solutions for two classes of fully nonlinear elliptic equations, Indiana Univ. Math. J. 39 (1990), 355-382. 
[49] X. J. WANG, A class of fully nonlinear elliptic equations and related functionals, Indiana Univ. Math. J. 43 (1994), 25-54.

JON JACOBSEN

Department of Mathematics

University of Utah

155 S. 1400 E.

Salt Lake City, UT 84112, USA

Current address: Department of Mathematics

Penn State University

University Park, PA 16802, USA

E-mail address: jacobsen@math.psu.edu 